\title{
2015 JAPAN Critical Limb Ischemia Database (JCLIMB) Annual Report
}

\author{
The Japanese Society for Vascular Surgery JCLIMB Committee, NCD JCLIMB Analytical Team
}

Since 2013, the Japanese Society for Vascular Surgery has started the project of nationwide registration and tracking database for patients with critical limb ischemia (CLI) who are treated by vascular surgeons. The purpose of this project is to clarify the current status of the medical practice for the patients with $\mathrm{CLI}$ to contribute to the improvement of the quality of medical care. This database, called JAPAN Critical Limb Ischemia Database (JCLIMB), was created on the National Clinical Database and collects data of patients' background, therapeutic measures, early results, and long term prognosis as long as five years after the initial treatment. The limbs managed conservatively are also registered in JCLIMB, together with those treated by surgery and/or endovascular treatment. In 2015, 1138 CLI limbs (male, 796 limbs [70\%]) were registered by 92 facilities. Arteriosclerosis obliterans has accounted for $98 \%$ of the pathogenesis of these limbs. In this manuscript, the background data and the early prognosis of the registered limbs are reported. (This is a translation of Jpn J Vasc Surg 2018; 27: 155-185.)

Keywords: critical limb ischemia, CLI, ASO, JCLIMB, NCD

\section{Introduction}

Recently, the number of patients with critical limb ischemia (CLI) who undergo medical care at clinical practice sites has been increasing. Approaches to improve the outcome of treatment for these patients are important and urgent issues. The Japanese Society for Vascular Surgery (JSVS) has initiated a nationwide CLI registration and tracking database project since 2013 to obtain epidemio-

Received: April 26, 2018; Accepted: April 26, 2018

Corresponding author: Tetsuro Miyata, MD, PhD. Japanese Society for Vascular Surgery, Odakyu Daiichi-Seimei Building 4F, 2-7-1 Nishi Shinjuku, Shinjuku-ku, Tokyo 163-0704, Japan

Tel: +81-3-5989-0991, Fax: +81-3-5324-0822

E-mail: tmiyata-tky@umin.ac.jp

This is a translation of Jpn J Vasc Surg 2018; 27: 155-185.

(cc) BY-NC-SA (C2018 The Editorial Committee of Annals of Vascular Diseases. This article is distributed under the terms of the Creative Commons Attribution License, which permits use, distribution, and reproduction in any medium, provided the credit of the original work, a link to the license, and indication of any change are properly given, and the original work is not used for commercial purposes. Remixed or transformed contributions must be distributed under the same license as the original. logical data on CLI that can be shared among the medical staff. The background of CLI limbs, contents of treatment, early outcome, and long term outcome until 5 years after surgery, including non-surgical limbs, are registered in this database. The database was named JAPAN Critical Limb Ischemia Database (JCLIMB) and established on the National Clinical Database (NCD). The primary objective of the JCLIMB project is to clarify the current status of CLI treatment performed by vascular surgeons in Japan, and feed it back to physicians at practice sites to improve the quality of medical care. The initial registration data and their tracking data one month after registration in 2013 and in 2014 has already been published. ${ }^{1,2)}$ This article reports the basic data registered in 2015 .

\section{JCLIMB}

Details of the registration, including the definition of CLI, have already been described in the 2013 annual report. ${ }^{1)}$ The followings are re-descriptions for confirmation.

CLI to be registered was defined according to TASC I $^{3}$ : chronic ischemic rest pain, ulcers or gangrene attributable to objectively proven arterial occlusive disease. The diagnosis of CLI should be confirmed by ankle pressure(AP) below $50 \mathrm{mmHg}$ or by toe pressure (TP) below $30 \mathrm{mmHg}$ in limbs with rest pain, and by AP below $70 \mathrm{mmHg}$ or by TP below $50 \mathrm{mmHg}$ in limbs with ulcer or gangrene.

The same limb can be registered in JCLIMB only once within a 5 -year tracking period. When the registered limb is treated in different periods or at different institutions, such data should be added only to the tracking items of each limb in JCLIMB, avoiding overlapping registration as a new limb with CLI. However, details of the procedure are registered each time in NCD apart from the registration in JCLIMB. On the other hand, the patient with bilateral CLI can be registered twice for each limb. Fixing JCLIMB data is done as follows, based on NCD regulations:

Initial registration data: Early April in the following year Tracking data early after treatment (1 month)/6 months after treatment: End of December in the following year Tracking data 1 year after treatment: End of December after 2 years 
Tracking data 2 years after treatment: End of December after 3 years

Tracking data 3 years after treatment: End of December after 4 years

Tracking data 4 years after treatment: End of December after 5 years

Tracking data 5 years after treatment: End of December after 6 years

As a general rule, the timing of tracking data registration is accepted within a \pm 2 -month range until 12 months after treatment, and within a \pm 3 -month range thereafter. Although the day for tracking data fixing is specified, it is made flexible because, in some limbs, follow-up data might be revealed later.

It was considered very difficult to make it obligatory for all the facilities participating in NCD to register CLI data since a great number of registration items in JCLIMB would put too much burden on them. Thus, facilities wishing to participate were recruited. In total, 92 facilities which registered CLI limbs in 2015 at the time of compiling in December 2016 are listed in the appendix.

Since JCLIMB is positioned as a registry study on NCD, the consent of patients for participation in the study and the ethical review of the study at the time of participation in NCD were adopted.

\section{Comments on the Aggregated Data in 2015}

The initial registration data in 2015 were fixed early April 2016, and the tracking data early after treatment (one month) were fixed on December 31, 2016. At the time of December 2016, 1138 limbs, those of 796 males (70\%) and 342 females (30\%), were registered by 92 facilities. All data and extracted data on arteriosclerosis obliterans (ASO) were collected according to the registered items. Since ASO accounted for $98 \%$ of all limbs, the overall and ASO data showed a similar tendency. In the comments, ASO data were presented in parentheses only when its figure was different from that of the overall data. In addition, because the WIfI classification of the Society for Vascular Surgery (SVS) was reported in 2014 (Tables 1-1-1 to 1-1-3),4) JCLIMB has made several changes and additions to the registered items to make WIfI classification possible since 2015 (Tables $\mathbf{1 - 2}-1$ to $\mathbf{1 - 2}-\mathbf{3}$ ). The total figure was not always consistent mostly due to missing values, and an explanation for each inconsistency was added.

\section{(1) Pretreatment patients' backgrounds}

Pretreatment patients' backgrounds are shown in Tables 2-1 to 2-6. Control of blood pressure was judged as good when it was below $140 / 90 \mathrm{mmHg}$ in the absence of diabetes and renal failure and below $130 / 80 \mathrm{mmHg}$ in the presence of these diseases. Control of diabetes was judged as good when hemoglobin A1c (HbA1c) was below 7.0\% (national glycohemoglobin standardization program [NGSP] value). Control of dyslipidemia was judged as good when low-density lipoprotein (LDL) was below 100 and $80 \mathrm{mg} / \mathrm{dL}$ in the absence and presence of other arteriosclerotic diseases, respectively. The presence of heart failure was judged clinically. The patient was regarded as having or having had heart failure when a past history of admission due to heart failure was present, clinical symptoms of heart failure were observed and confirmed on echocardiography, or cardiac function was clearly reduced on echocardiography although no clinical symptom was present. Renal dysfunction was graded following the new chronic kidney disease severity classification of the "Clinical Practice Guidebook for Diagnosis and Treatment of Chronic Kidney Disease 2012" ${ }^{5)}$ : Renal dysfunction was absent when the estimated glomerular filtration rate (eGFR) $\left(\mathrm{mL} / \mathrm{min} / 1.73 \mathrm{~m}^{2}\right)$ was 60 or higher, and it was graded as G3a, G3b, G4, and G5 when eGFR was 45-59, $30-44,15-29$, and below 15 , respectively. eGFR below 15 in hemodialysis patients was graded as G5D.

The causes of the arterial occlusion of the limb were ASO in 1114 (98\%) limbs, thromboangiitis obliterans (TAO) in 10, vasculitis (Takayasu's arteritis, collagen disease, Behçet's disease, and fibromuscular dysplasia excluding TAO) in eight, and others in six. Comorbidities of the patients consisted of diabetes in $67 \%(68 \%)$ of the limbs, hypertension in $73 \%(74 \%)$, dyslipidemia in $38 \%(39 \%)$, ischemic heart disease in $43 \%$ (44\%), cerebrovascular disease in $22 \%$, dialysis for renal failure in $43 \%(44 \%)$, past medical history of malignant neoplasm or that being treated in $8 \%(9 \%)$, and arterial occlusive lesions in the opposite limb in $75 \%$ (76\%).

The problems and considerations on these spreadsheets are described below. In Table 2-4, describing the medical history of malignant neoplasm, the sum of the numbers in the column with the history of malignant neoplasm ("history of cancer", "under treatment", and "unknown") is larger than that of the numbers in the column with the sites of malignant neoplasm, in the row of limbs of Rutherford 5. As there might be duplicated cancers, the total number of sites of malignant neoplasm should be the same or more than that in the column with the history of malignant neoplasm. This is due to the following reasons. When "unknown" is selected about the information of malignancy, the input screen for the part of the malignancy is not displayed. As a result, the information on the site of malignancy was not input in five "unknown" limbs. In addition, because there were four limbs with duplicated cancer, the total number of sites of malignant neoplasm decreased by one as a whole. 


\section{(2) Conditions of limb ischemia}

The pretreatment conditions of limb ischemia are shown in Tables 3-1 to 3-6. Regarding the walking function (Taylor classification), ${ }^{6)}$ patients with the ability to walk outdoors or indoors independently, including with a cane, were regarded as "ambulatory", and those unable to walk but able to stand on their own legs during transfer from the bed to a wheel chair were designated as "ambulatory/ homebound."

Regarding the state of local tissue defect (Texas University Classification), ${ }^{7)}$ the most severe lesion being the main target of treatment was evaluated. Skin perfusion pressure (SPP) was measured on the foot (base of the toe, dorsum of the foot, or sole) and a lower value was adopted. In addition, in order to perform WIfI classification, the sites of ulcer and gangrene were registered separately. Although SPP is widely used as an objective index to evaluate ischemia in Japan, ischemic grading criteria using SPP is not shown in WIfI classification, in which TP is given top priority. Therefore, in JCLIMB, the SPP value was converted to TP using the conversion equation $\mathrm{TP}=0.6853 \mathrm{SPP}+14.48$ from the correlation data of SPP and TP reported in Japan, ${ }^{8)}$ and applied for WIfI ischemic grading (Table 1-2-2).

The lesion was regarded as infected when it showed two or more of the following findings: local swelling or induration, erythema $>0.5 \mathrm{~cm}$ around the ulcer, local tenderness or pain, local warmth, purulent discharge (thick, opaque to white, or sanguineous secretion). In addition, local infections involving only the skin and the subcutaneous tissue and those involving structures deeper than the skin and subcutaneous tissues were registered separately. Local infections involving only the skin and the subcutaneous tissue were differentiated according to the size of the erythema around the ulcer, $\leqq 2$ or $>2 \mathrm{~cm}$.

Systemic inflammatory response syndrome (SIRS), indicating systemic infection, was manifested by two or more of the following signs: temperature $>38^{\circ} \mathrm{C}$ or $<36^{\circ} \mathrm{C}$, heart rate $>90$ beats $/ \mathrm{min}$, respiratory rate $>20$ breaths/min or $\mathrm{PaCO}_{2}<32 \mathrm{mmHg}$, white blood cell count $>12,000$ or $<4000 \mathrm{cu} / \mathrm{mm}$ or $10 \%$ immature (band) forms. The arteries in the ankle joint region were classified as foot arteries.

Pretreatment ambulatory function was ambulatory in $55 \%$ of the limbs, ambulatory/homebound in $24 \%$, and non-ambulatory in $21 \%$. On Rutherford classification (R), 9) limbs with categories R4, R5, and R6 accounted for $21 \%, 64 \%$, and $15 \%$ of the limbs, respectively. The median ankle brachial index (ABI), the toe brachial index (TBI), and the SPP of the measured limbs was $0.61(0.60)$, $0.27(0.26)$, and $22 \mathrm{mmHg}$, respectively. The occlusive legion was located in the aortoiliac artery in $16 \%$ of the limbs, in the femoropopliteal artery in $41 \%(42 \%)$, and in the crural or foot artery in $43 \%$.

We were able to apply the WIfI classification with sufficient data to 859 limbs (841 limbs). On the WIfI classification, limbs with the stages $1,2,3$, and 4 accounted for $10 \%, 23 \%, 26 \%(27 \%)$, and $40 \%(41 \%)$ of the limbs, respectively.

The problems and considerations on these spreadsheets are described below. In Table 3-3, the total number of limbs in TASCII classification differed compared to the number in each column of the site of occlusion. In "aortoiliac" lesion, decreased number of that in TASCII classification may have been due to input omission. In "femoropopliteal" lesion, increased number of that in TASCII may have been due to inclusion of crural lesions.

In Table 3-6, there were 113 limbs (110 limbs) which classified to Wound grade 3 (W3; extensive ulcer/gangrene) in WIfI classification in the row of limbs of R5 (small-range tissue defect). Such results might have been obtained when there was a deep ulcer or gangrene in the heel, even if the wound was not extensive. In addition, any size of gangrene in parts other than toes, even if it was small, could be classified to W3.

In Table 3-6, 81 limbs (77 limbs) were registered as Ischemic grade 0 in WIfI classification. By definition, a limb with Ischemic grade 0 has a TP of $60 \mathrm{mmHg}$ or more (SPP $66 \mathrm{mmHg}$ or more in JCLIMB) or AP higher than $100 \mathrm{mmHg}$, or if arterial calcification precludes reliable AP or TP measurements, $\mathrm{TcPO}_{2} 60 \mathrm{mmHg}$, or more (Table 1-1-2). There should be no limb with Ischemic grade 0 since CLI to be registered in JCLIMB is defined according to TASC II. There is a possibility that the limbs clinically judged to be CLI were registered irrespective of the objective ischemic index, although details are unknown.

In Table 3-6, there were 24 limbs (23 limbs) in which infection was confirmed in R4 limbs, despite the absence of a local wound by definition of R4. The details are unclear whether the limb showed the symptoms of cellulitis without any wound or there was a small wound somewhere (in this situation it might be better to classify the limbs in R5).

In Table 3-6, because the data on ischemic grade were registered in only 859 limbs (841 limbs) among 1138 limbs (1114 limbs), WIfI classification could be implemented for these 859 limbs (841 limbs). When rechecking the remaining 279 limbs (273 limbs), the data on TBI, SPP, or ABI in these limbs were registered as unmeasurable or unmeasured. It seems to be unlikely that these ischemic indexes could not be measured in these limbs due to the extensive gangrene because 85 limbs with R4, 138 limbs (133 limbs) with R5 and 56 limbs (55 limbs) with R6 were included in this unmeasurable or unmeasured group. There is a possibility that the limbs clinically judged to be CLI were registered without their objective ischemic index. 


\section{(3) Treatment}

Tables 4-1 to 4-6 show the data on the treatment of CLI. Revascularizations of the affected limbs were performed in $96 \%$ of the registered limbs, and primary major amputations were performed in $1.9 \%$ of the registered limbs. Among the procedures of surgical reconstruction, distal bypass, which is a bypass to the crural or foot artery, accounted for $46 \%(45 \%)$. Endovascular treatment (EVT), including EVT alone and hybrid treatment with surgical reconstruction, accounted for $58 \%(59 \%)$ of the total revascularization procedures. EVT applied to the crural or foot artery accounted for $39 \%$ of the total EVT.

The problems and considerations on these spreadsheets are described below. Table 4-3, in the column of "vein usage" described how the autologous veins were used when they were selected as vascular conduits. The sum of the number in the column with vein usage; "in-situ," "non-reversed," "reversed" and "spliced," is larger than the sum of the number in the column of vein in vascular prosthesis. It is speculated to be caused by selecting multiple vein usage for arterial reconstruction of a limb since it is permitted to select more than one vein usage.

Table 4-6 summarizes the vascular grafts used for the infra-inguinal arterial reconstruction. For example, the total number of femoral-above knee popliteal artery bypass was 109 (107), higher than 102 (100), the number of actual applications in Table 4-2. It may have reflected the content of other procedures because the bypass procedure can be simultaneously applied with other procedures. Multiple procedures can be selected at the same time for lower limb arterial reconstruction. This is also the reason for the presence of "unused."

\section{(4) Outcomes early (one month) after treatment}

Tables 5-1 to 5-8 show the outcomes early (one month) after treatment. At the time of summary count at the end of December 2016, follow-up data one month after treatment were obtained in 837 limbs (74\%) including 816 limbs $(73 \%)$ with ASO. There were 36 limbs with nonarterial reconstruction. Data were collected according to the severity of the local conditions of the limb (Rutherford classification) and treatment measures (EVT alone or surgical reconstruction with/without EVT). The mortality was $2.6 \%(2.7 \%)$ in the whole series, and $2.3 \%$ and $3.1 \%$ $(3.2 \%)$ treated by EVT alone and by surgical reconstruction with/without EVT, respectively. The most common cause of death was cardiac disease, accounting for $27 \%$ of all deaths.

Postoperative complications were cardiac disease in $2.1 \%(2.2 \%)$, cerebrovascular disease in $0.8 \%$, pneumonia in $1.6 \%(1.7 \%)$, and wound complication in $5.0 \%$ $(4.8 \%)$. Complications at the puncture site were noted in $0.5 \%$ of limbs treated by EVT. The median ABI and SPP of the measured limbs were 0.89 and $43 \mathrm{mmHg}$, respectively.

Stenosis, occlusion, and infection occurred after revascularization by EVT in 9.9\% $(9.7 \%)$ and by surgical reconstruction in $8.3 \%(7.6 \%)$. Secondary major amputation was performed in $4.6 \%(4.3 \%)$ of the limbs.

When ambulatory function at discharge was compared with that before surgery, the rate of patients with ambulatory changed from $55 \%$ to $53 \%(52 \%)$, ambulatory/ homebound from $24 \%$ to $23 \%$, and nonambulatory from $21 \%$ to $24 \%(25 \%)$.

The problems, comments, and considerations on these spreadsheets are described below. Among 36 limbs of survivors with non-arterial reconstruction (Table 5-1), 4 limbs underwent primary major amputation and were counted in the column of perioperative complications in the row of limbs with non-arterial reconstruction (Table 5-2). Therefore, 36 limbs of survivors with non-arterial reconstruction comprised 4 limbs with primary major amputation and 32 limbs with conservative treatment.

The number of limbs of survivors with EVT was 344 (339 limbs) (Table 5-1), which was 5 limbs higher than the sum of the number in the column of minor reintervention or major reintervention in the row of limbs with EVT; 339 limbs (334 limbs) (Table 5-6). Four of these 5 limbs underwent major amputation after EVT, and reintervention was not performed. The information related to reintervention on the remaining one limb was missing.

The number of limbs of survivors with surgical reconstruction was 435 (419 limbs) (Table 5-1), which was one limb higher than the sum of the number in the column of minor reintervention or major reintervention in the row of limbs with surgical reconstruction; 434 limbs (418 limbs) (Table 5-6). This one limb also underwent major amputation after surgical reconstruction, and reintervention was not performed.

In Table 5-6, the sum of the number of limbs in the column of "major amputation" was expected to be 811 limbs (790 limbs); the limbs of survivors without major amputation comprised 32 limbs with conservative treatment, 344 limbs (339 limbs) with EVT, and 435 limbs (419 limbs) with surgical reconstruction. But the actual sum of the number of limbs in the column of major amputation was 808 (787), indicating 3 limbs fewer than expected. This was due to unregistered limbs with EVT; the sum of the number of limbs in the row of EVT was 341 (336), indicating that 3 limbs were unregistered.

In addition to the above, there were some parts where the total number does not match in Tables 5-1 to 5-8. It is estimated to be due to several items with multiple choice or missing values. 


\section{Conclusions}

The devoted contribution of vascular surgeons in the participating facilities to register a sufficient amount of detailed data during busy clinical practice has been gradually clarifying the current status of CLI treatment in Japan; data on CLI in 2015 were clarified, after those in 2013 and 2014. The JCLIMB Committee is planning to continue publishing an annual report. Facilities can newly participate in JCLIMB at any time, and clinical studies utilizing these data will also be performed under specific conditions. Please contact the secretariat of the JSVS for details.

In the future, JCLIMB is designed so as to be extended to a system which physicians in departments other than vascular surgery will be able to register, track, and analyze CLI, aiming at establishing a nationwide CLI database in Japan.

\section{Participant Facilities (92 facilities in the order of the Japanese syllabary by area, corporate names are omitted as a rule)}

Department of Vascular Surgery, Asahikawa Medical University Hospital

Department of Cardiovascular Surgery, National Hospital Organization Obihiro Hospital

Department of Cardiovascular Surgery, National Hospital Organization Hokkaido Medical Center

Department of Cardiovascular Surgery, Steel Memorial Muroran Hospital

Department of Cardiovascular Surgery, Nayoro City General Hospital

Department of Surgery, Iwate Prefectural Iwai Hospital

Department of Surgery, Iwate Prefectural Isawa Hospital

Department of Cardiovascular Surgery, Iwate Prefectural Central Hospital

Department of Surgery, Iwate Prefectural Chubu Hospital Department of Surgery, JR Sendai Hospital

Department of Surgery and Cardiovascular Surgery, Sendai City Hospital

Department of Transplantation, Reconstruction and Endoscopic Surgery, Tohoku University Hospital

Department of Cardiovascular Surgery, Southern TOHOKU General Hospital

Department of Thoracic and Cardiovascular Surgery, Hirosaki University Hospital

Department of Vascular Surgery, Morioka Yuai Hospital

Department of Cardiovascular Surgery, Akita Kouseiren Yurikumiai General Hospital

Department of Cardiovascular Surgery, Itabashi Chuo Medical Center

Department of Vascular Surgery, Ibaraki Prefectural Cen- tral Hospital

Department of Cardiovascular Surgery, IMS Tokyo Katsushika General Hospital

Department of Vascular Surgery, Edogawa Hospital

Department of Vascular Surgery, Kawasaki Municipal Hospital

Department of Cardiovascular Surgery, Kyorin University Hospital

Department of Surgery, Keio University Hospital

Department of Surgery, Tokyo Metropolitan Health and Medical Treatment Corporation, Okubo Hospital

Department of Vascular Surgery, International University of Health and Welfare Hospital

Department of Vascular Surgery, International University of Health and Welfare, Mita Hospital

Department of Vascular Surgery, Saiseikai Kawaguchi General Hospital

Department of Vascular Surgery, Saiseikai Yokohamashi Tobu Hospital

Department of Vascular Surgery, Saitama Medical Center

Department of Surgery, Saitama City Hospital

Department of Cardiovascular Surgery, Saitama Medical Center, Jichi Medical University

Department of Surgery, Shonankamakura General Hospital

Department of Cardiovascular Surgery, St. Marianna University School of Medicine

Department of Cardiovascular Surgery, Shimada General Hospital

Department of Cardiovascular Surgery, Chiba Central Medical Center

Department of Vascular Surgery, Tokyo Medical and Dental University

Department of Cardiovascular Surgery, Tokyo Medical University Hachioji Medical Center

Department of Cardiovascular Surgery, Tokyo Medical University Hospital

Department of Vascular Surgery, The Jikei University Kashiwa Hospital

Department of Vascular Surgery, The Jikei University Hospital

Department of Cardiovascular Surgery, Tokyo Women's Medical University Medical Center East

Department of Vascular Surgery, The University of Tokyo Hospital

Department of Cardiovascular Surgery, Tokyo Rinkai Hospital

Department of Vascular Surgery, Tomei Atsugi Hospital

Department of Cardiovascular Surgery, Tokorozawa Meisei Hospital

Department of Cardiac and Vascular Surgery, Dokkyo Medical University Nikko Medical Center

Department of Cardiac and Vascular Surgery, Dokkyo 
Medical University Hospital

Department of Cardiovascular Surgery, National Defense Medical College Hospital

Department of Cardiovascular Surgery, Yokosuka General Hospital UWAMACHI

Department of Vascular Surgery, Aichi Medical University Hospital

Department of Vascular Surgery, Ichinomiya Municipal Hospital

Department of Cardiovascular Surgery, National Hospital Organization, Kanazawa Medical Center

Department of Vascular Surgery, Japanese Red Cross Shizuoka Hospital

Department of Vascular Surgery, Japanese Red Cross Nagoya Daiichi Hospital

Department of Vascular Surgery, Nagoya University Hospital

Department of Cardiovascular Surgery (Vascular Surgery), Osaka International Cancer Institute

Department of Vascular Surgery, Osaka Rosai Hospital

Department of Cardiovascular Surgery, Tsukazaki Hospital

Department of Vascular Surgery, Kansai Medical University Medical Center

Department of Cardiovascular Surgery, Kobe University Hospital

Department of Cardiovascular Surgery, Toyonaka Municipal Hospital

Department of Surgery, Shinsuma General Hospital

Department of Vascular Surgery, Soryukai Inoue Hospital

Department of Cardiovascular Surgery, Hashimoto $\mathrm{Mu}-$ nicipal Hospital

Department of Thoracic and Cardiovascular Surgery, Wakayama Medical University Hospital

Department of Cardiovascular Surgery, Ehime Prefectural Central Hospital

Department of Cardiovascular Surgery, Okayama University Hospital

Department of Cardiovascular Surgery, Kawasaki Medical School Hospital

Department of Cardiovascular Surgery, Kochi Health Sciences Center

Department of Cardiovascular Surgery, Kochi University Hospital

Department of Cardiovascular Surgery, National Hospital Organization Higashihiroshima Medical Center

Department of Vascular Surgery, Saiseikai Yamaguchi General Hospital

Department of Cardiovascular Surgery, Tottori Prefectural Central Hospital

Department of Cardiovascular Surgery, The Sakakibara Heart Institute of Okayama

Department of Cardiovascular and Respiratory Surgery,
Hiroshima Prefectural Hospital

Department of Surgery, Hiroshima Red Cross Hospital \& Atomic-bomb Survivors Hospital

Department of Cardiovascular Surgery, Hiroshima University Hospital

Department of Cardiovascular Surgery, Matsuyama Shimin Hospital

Department of Vascular Surgery, Matsuyama Red Cross Hospital

Department of Vascular Surgery, Yamaguchi University Hospital

Department of Cardiovascular Surgery, Oita Oka Hospital

Department of Vascular Surgery, Kyushu University Hospital

Department of Vascular Surgery, Kumamoto Rehabilitation Hospital.

Cardiovascular Surgery, Kurume University Hospital

Department of Vascular Surgery, Kokura Memorial Hospital

Department of Vascular Surgery, National Hospital Organization Kyushu Medical Center

Department of Surgery, Saiseikai Karatsu Hospital

Department of Surgery, Saiseikai Fukuoka General Hospital

Department of Cardiovascular Surgery, Saga-ken Medical Center, Koseikan

Department of Cardiovascular Surgery, Sasebo Chuo Hospital

Department of Vascular Surgery, Steel Memorial Yawata Hospital

Department of Vascular Surgery, Fukuoka City Hospital

\section{JCLIMB Committee, NCD JCLIMB Ana- lytical Team}

(1) JCLIMB Steering Committee

Tetsuro Miyata (Chairman), Masamitsu Endo, Nobuyoshi Azuma, Takao Ohki, Kimihiro Komori, Osamu Sato, and Shunya Shindo

\section{(2) CLIMB Practices Committee}

Masamitsu Endo (Chairman), Tetsuro Miyata, Atsuhisa Ishida, Yuichi Izumi, Yoshinori Inoue, Hisashi Uchida, Koji Kurosawa, Hiroyoshi Komai, Kunihiro Shigematsu, Takashi Shibuya, Ikuo Sugimoto, Masayuki Sugimoto, Juno Deguchi, Naomichi Nishikimi, Katsuyuki Hoshina, Hideaki Maeda, Shinsuke Mii, Hirofumi Midorikawa, Terutoshi Yamaoka, Hiroya Yamashita, and Masahiro Yunoki

(3) NCD JCLIMB Analytical Team

Arata Takahashi and Hiroaki Miyata 


\section{Disclosure Statement}

The authors have no conflict of interest.

\section{Additional Remarks}

The original Annual Report was published in the Japanese Journal of Vascular Surgery Vol. 27 (2018) No. 3; however, errors in tables were detected after the publication. The erratum was published in the same volume. This translation reflects the corrections.

\section{References}

1) Japanese Society for Vascular Surgery JCLIMB Committee, NCD JCLIMB Analytical Team. 2013 JAPAN Critical Limb Ischemia Database (JCLIMB) Annual Report. Ann Vasc Dis 2016; 9: 356-73.

2) Japanese Society for Vascular Surgery JCLIMB Committee, NCD JCLIMB Analytical Team. 2014 JAPAN Critical Limb Ischemia Database (JCLIMB) Annual Report. Ann Vasc Dis 2016; 9: 374-91.

3) Norgren L, Hiatt WR, Dormandy JA, et al. Inter-society consensus for the management of peripheral arterial disease (TASC II). J Vasc Surg 2007; 45: S5-67.

4) Mills JL Sr, Conte MS, Armstrong DG, et al. The Society for Vascular Surgery Lower Extremity Threatened Limb Classification System: risk stratification based on wound, ischemia, and foot infection (WIfI). J Vasc Surg 2014; 59: 220-34.

5) Japanese Society of Nephrology. Clinical Practice Guidebook for Diagnosis and Treatment of Chronic Kidney Disease 2012. Tokyo: Tokyo Igakusya; 2012. (in Japanese)

6) Taylor SM, Kalbaugh CA, Gray BH, et al. The LEGS score: a proposed grading system to direct treatment of chronic lower extremity ischemia. Ann Surg 2003; 237: 812-9.

7) Armstrong DG, Lavery LA, Harkless LB. Validation of a diabetic wound classification system. The contribution of depth, infection, and ischemia to risk of amputation. Diabetes Care 1998; 21: 855-9.

8) Yamada T, Ohta T, Ishibashi $\mathrm{H}$, et al. Clinical reliability and utility of skin perfusion pressure measurement in ischemic limbs - comparison with other noninvasive diagnostic methods. J Vasc Surg 2008; 47: 318-23.

9) Rutherford RB, Baker JD, Ernst C, et al. Recommended standards for reports dealing with lower extremity ischemia: revised version. J Vasc Surg 1997; 26: 517-38. 


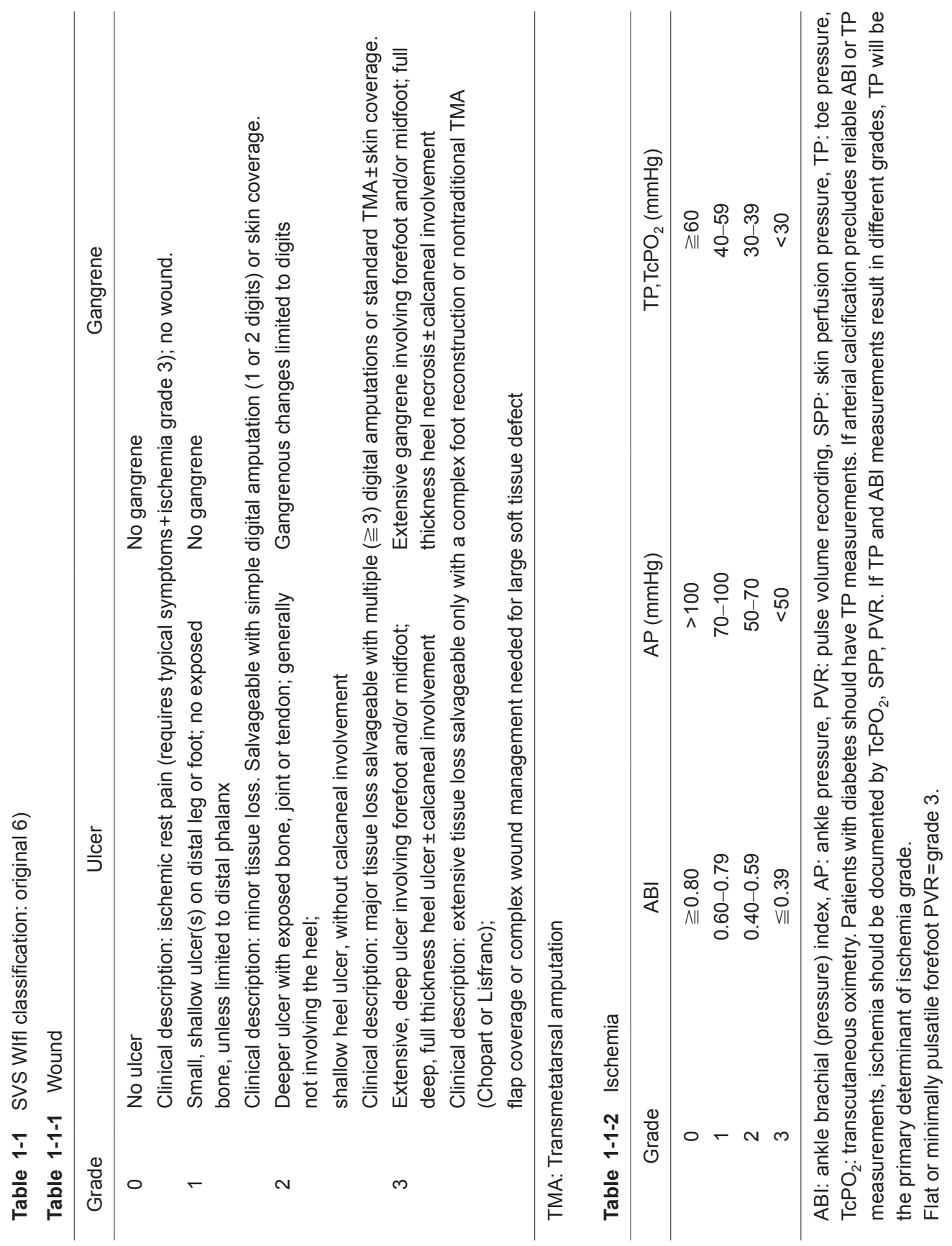




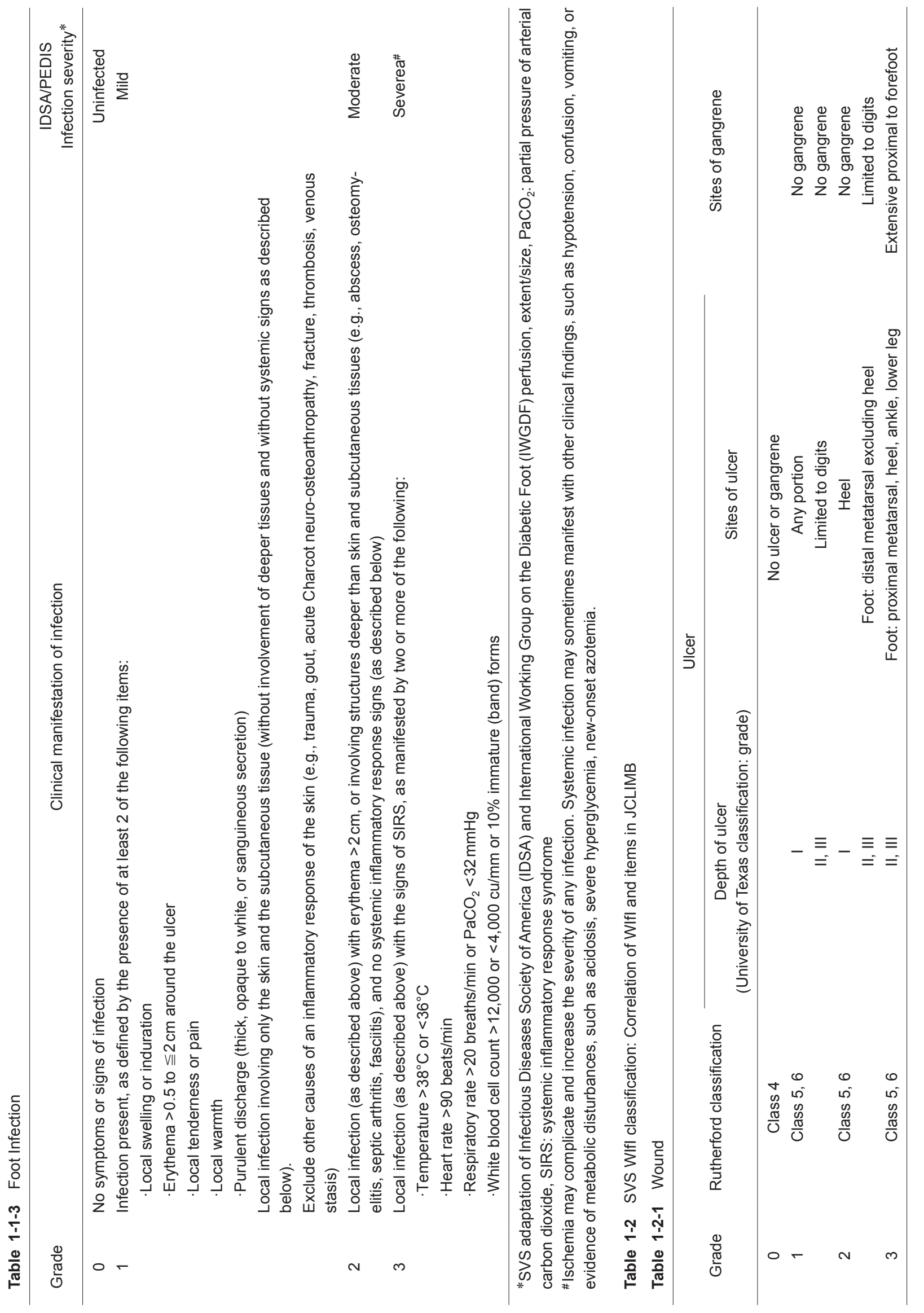




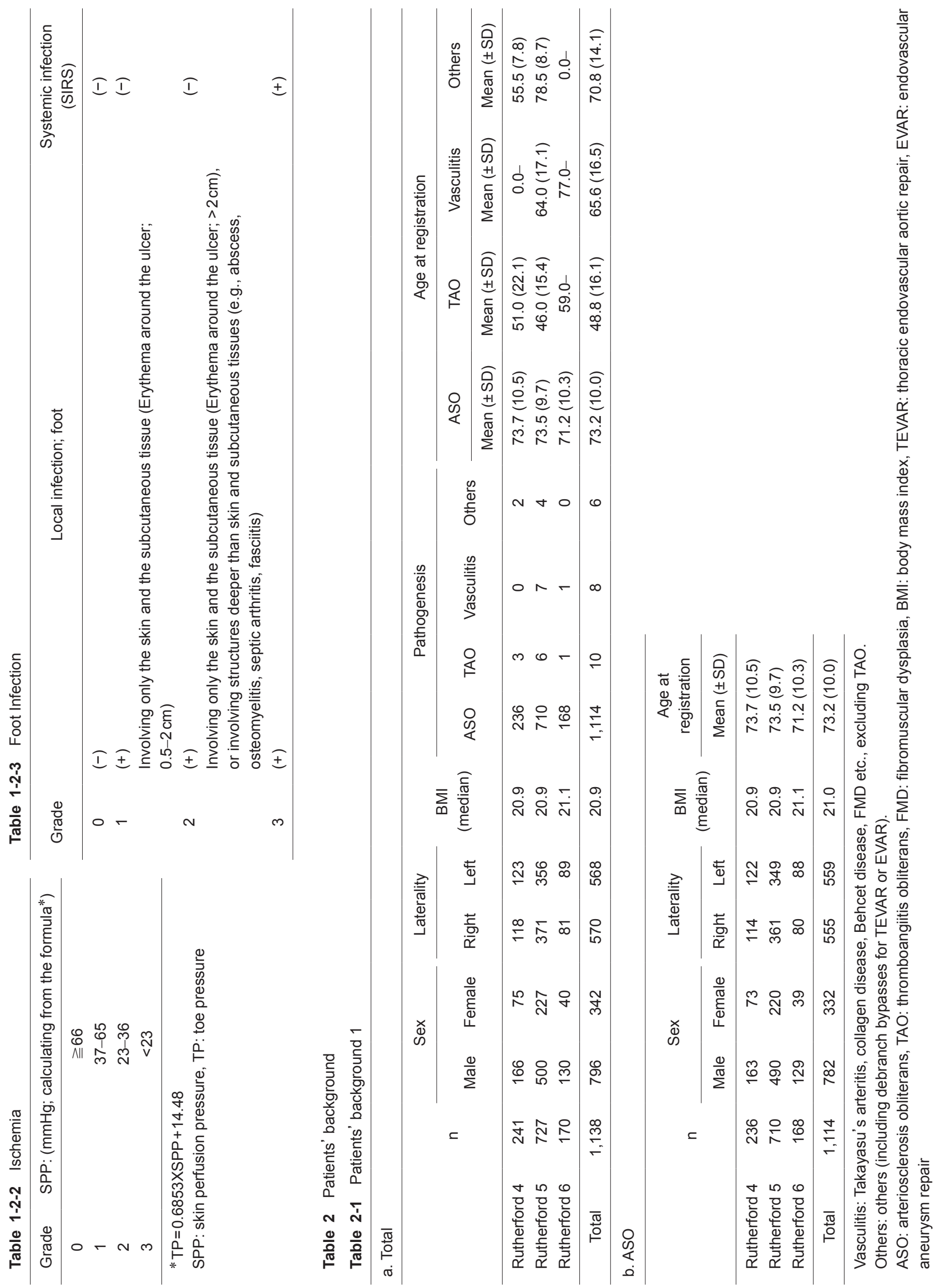




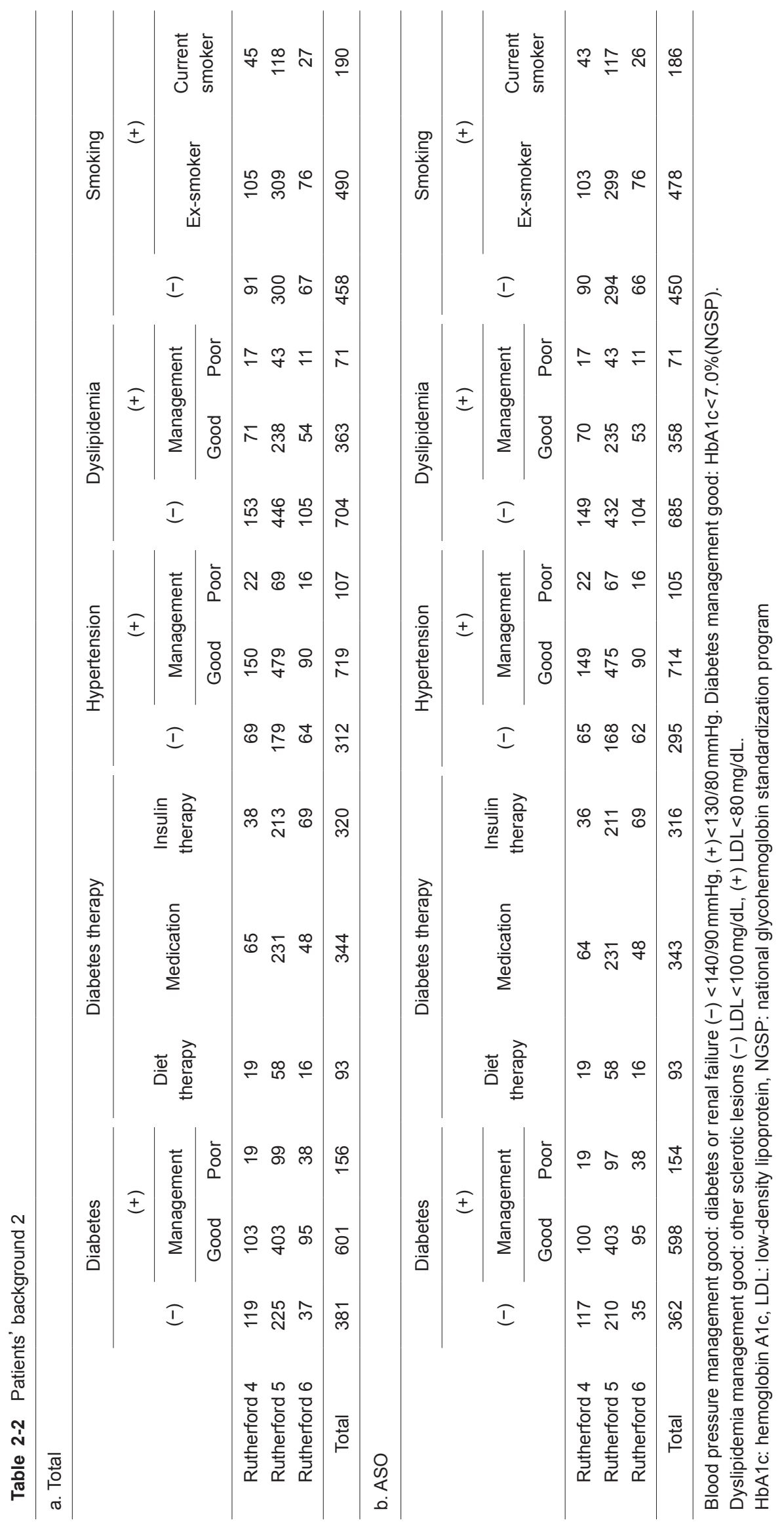




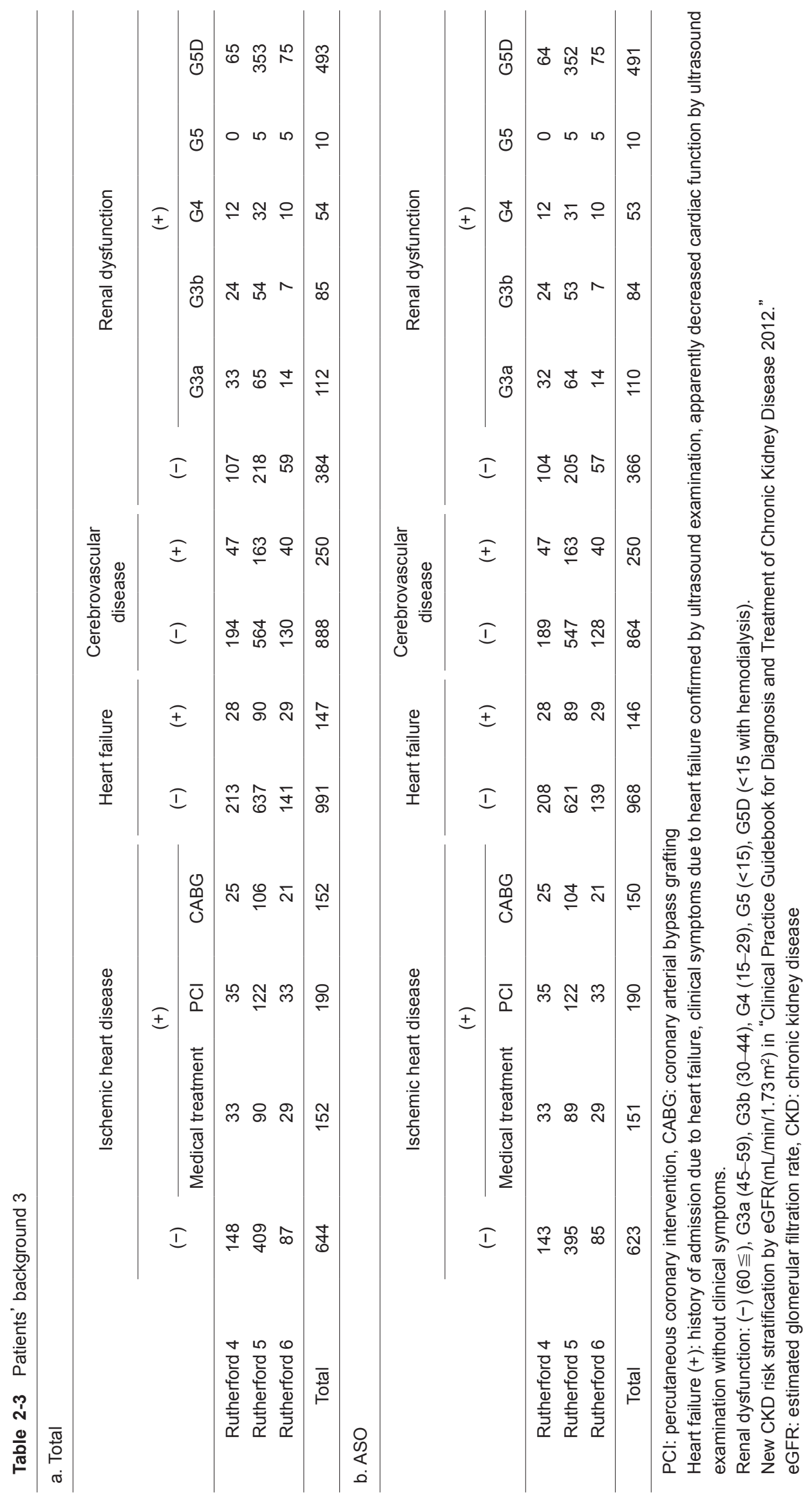




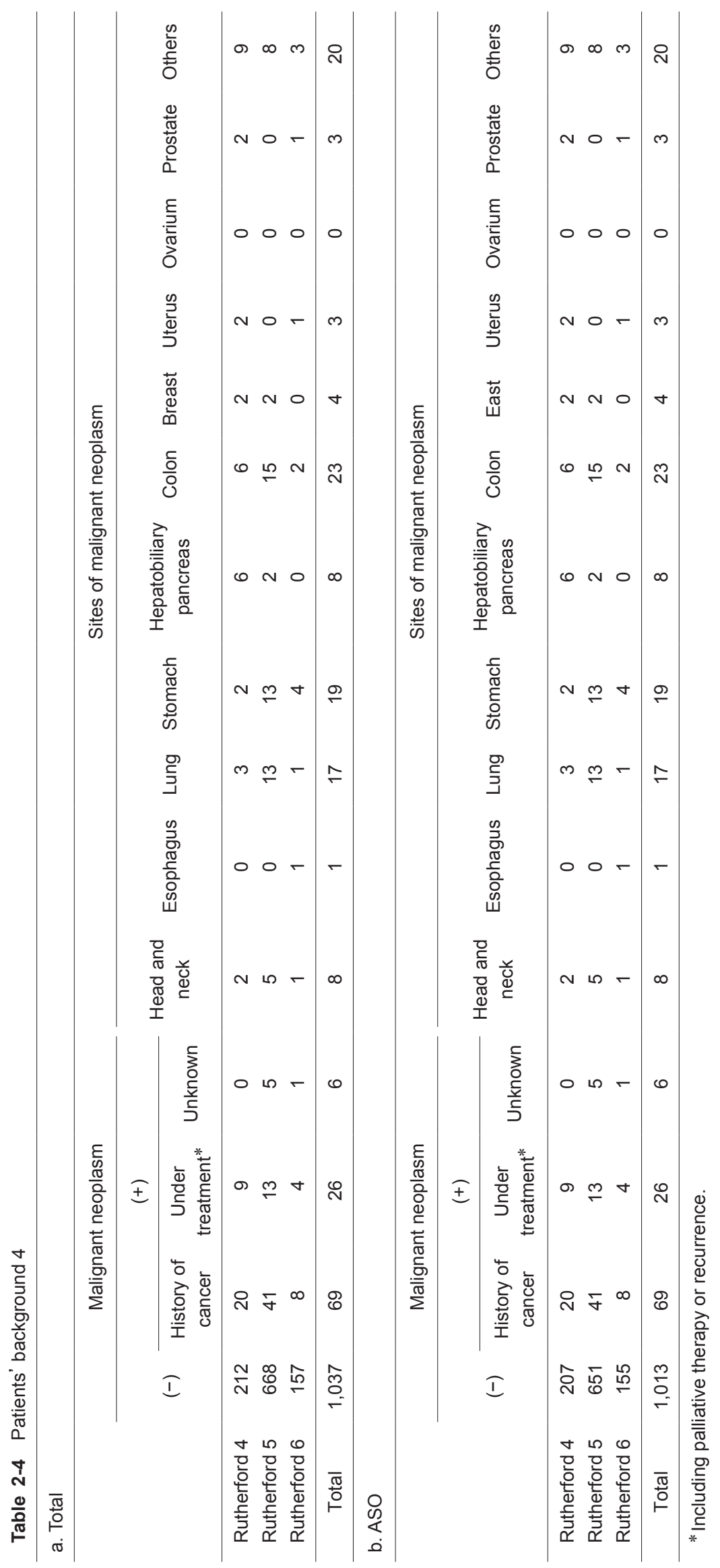




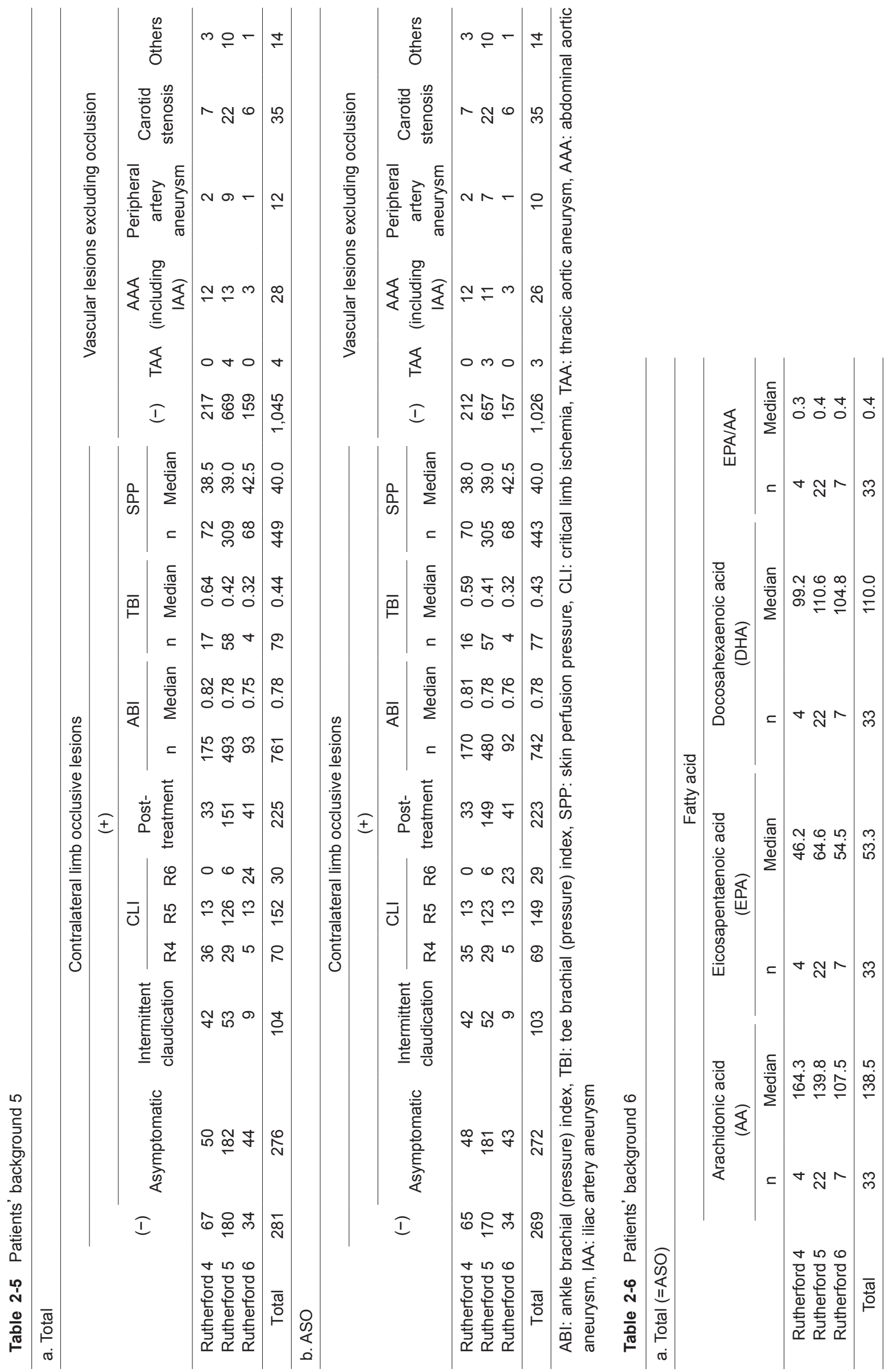




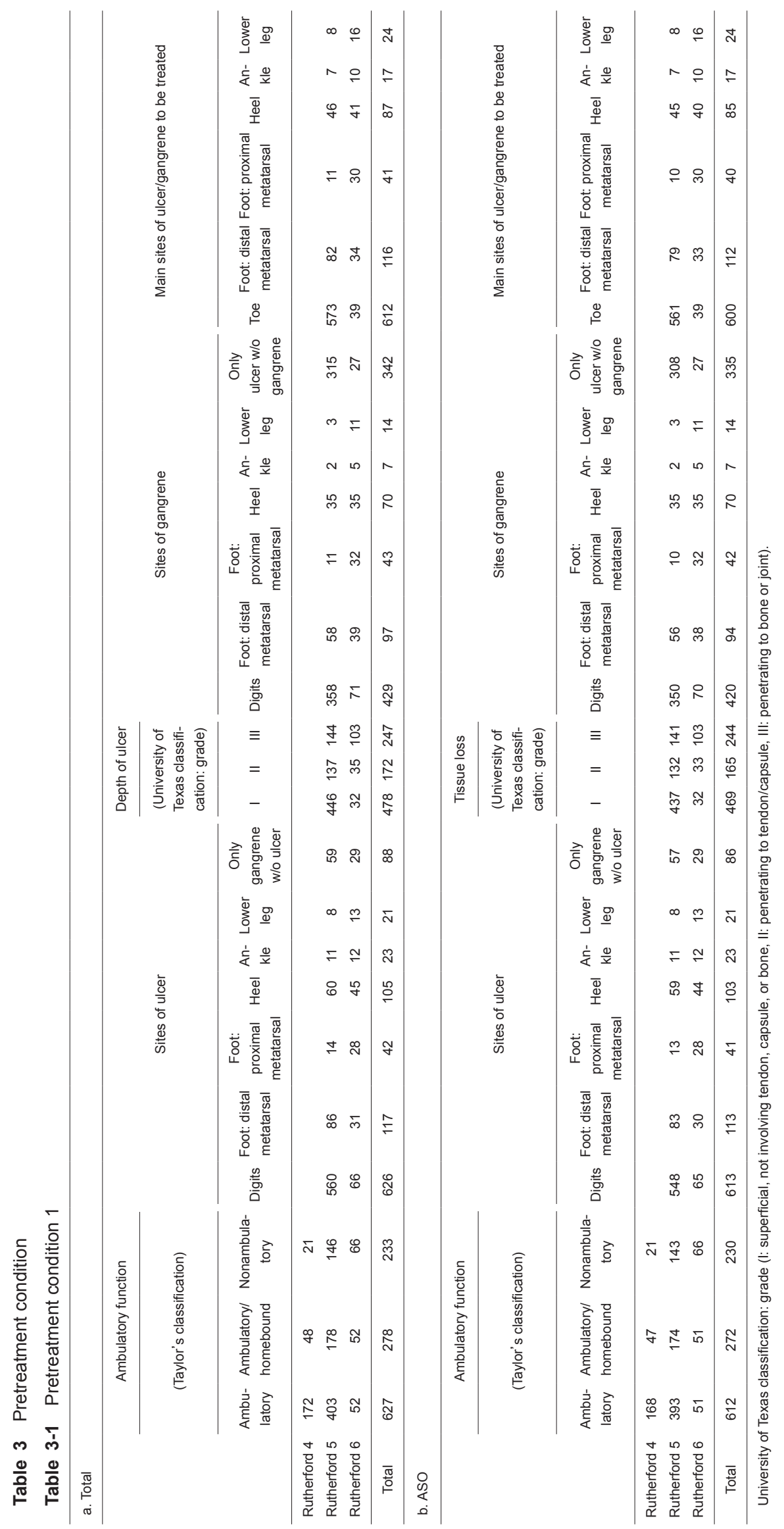




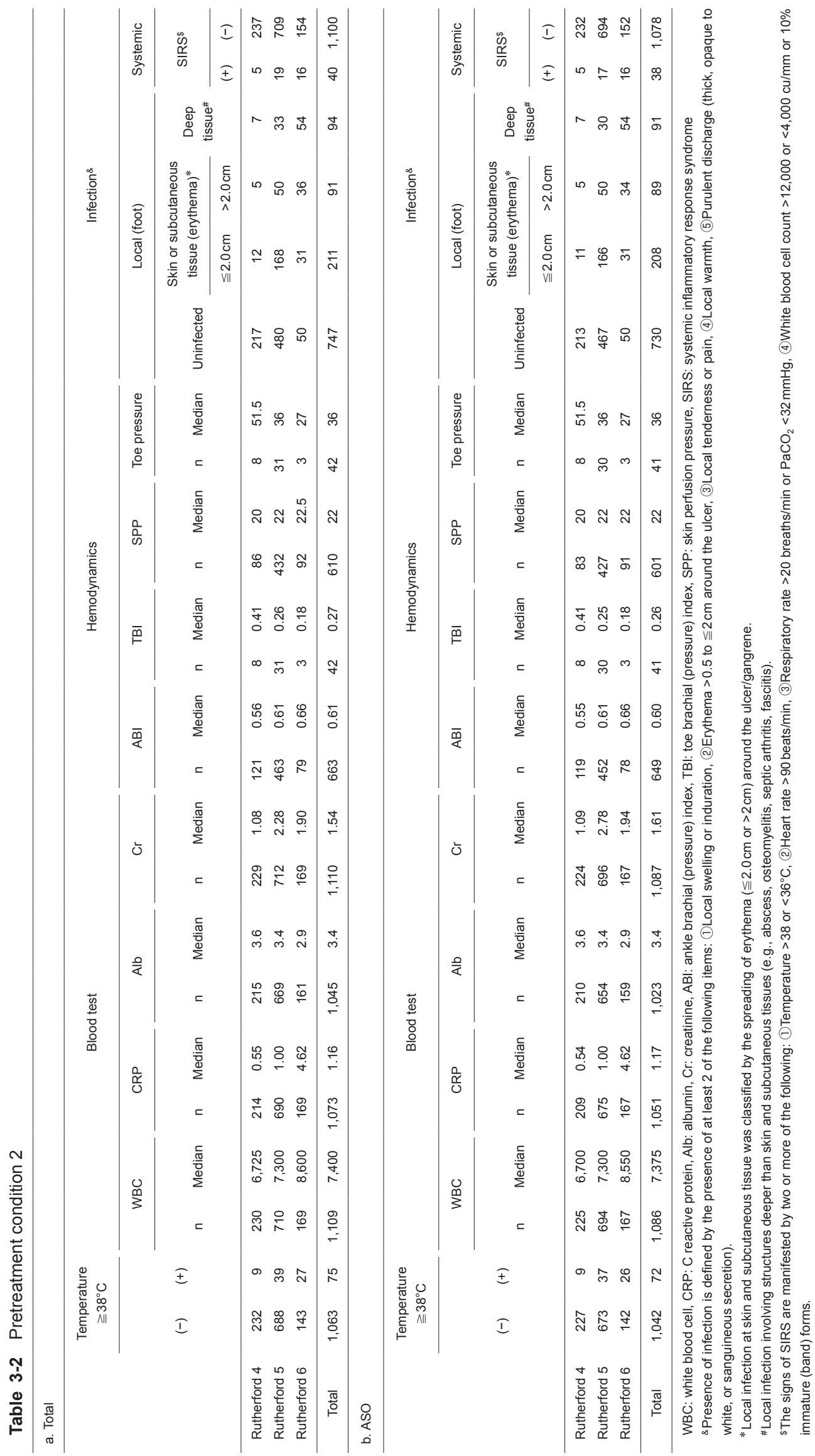




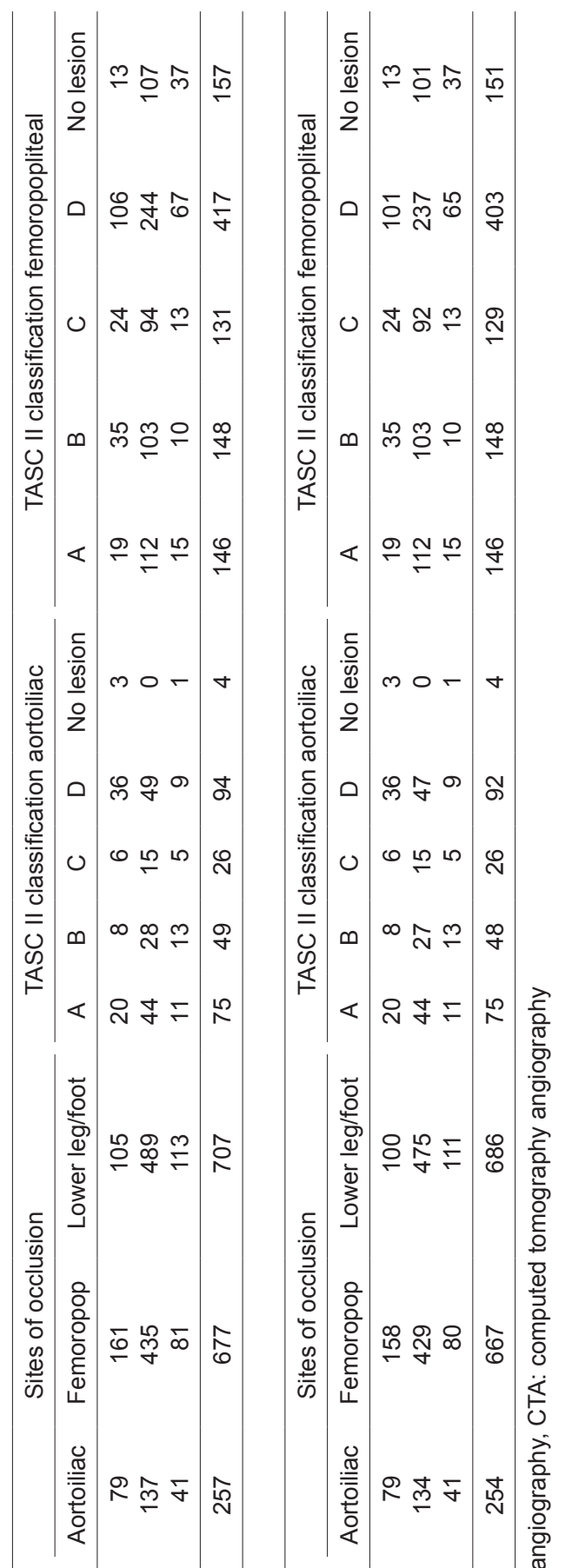

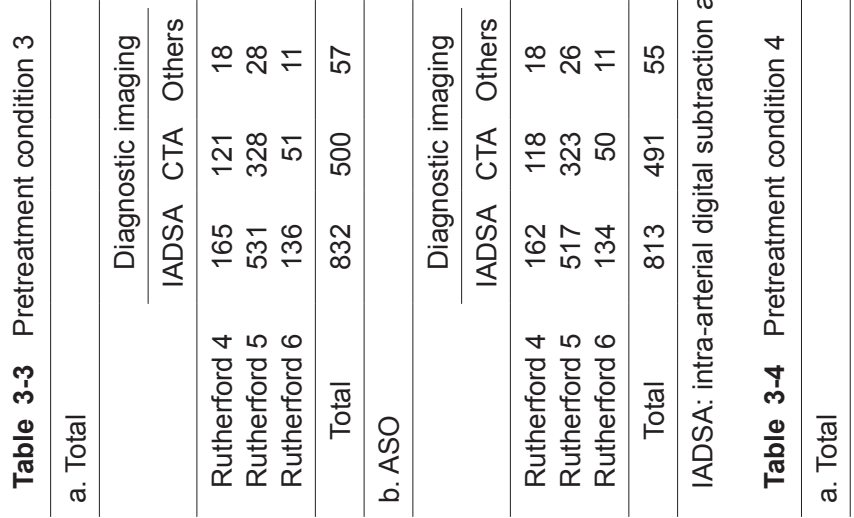
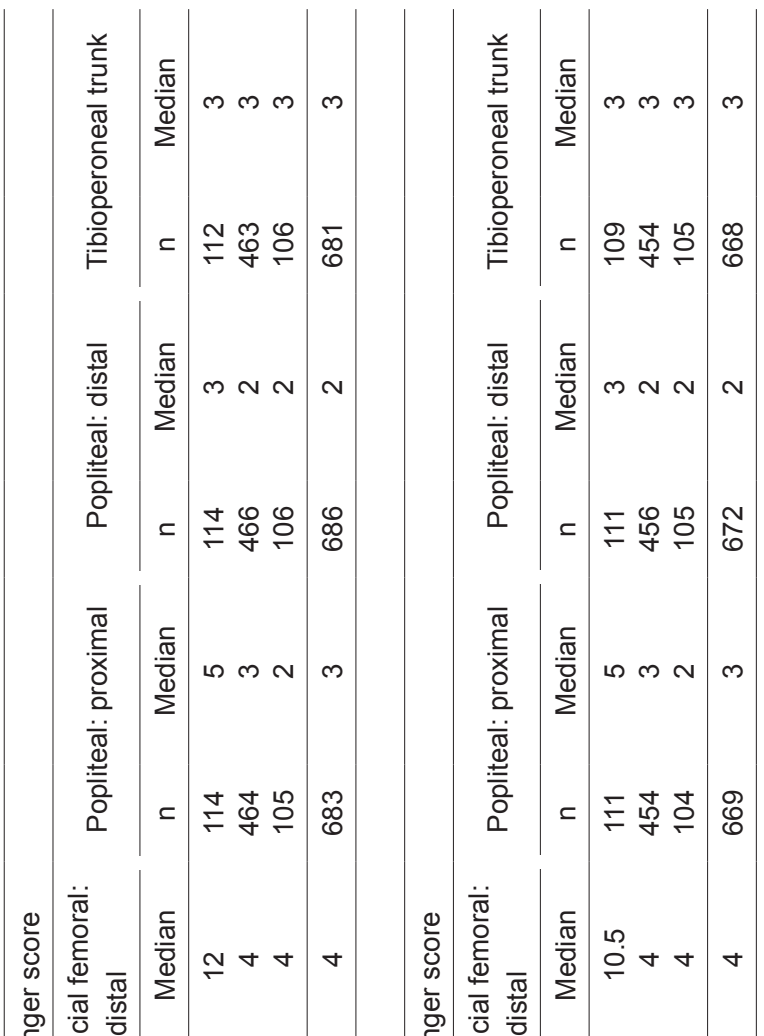

言
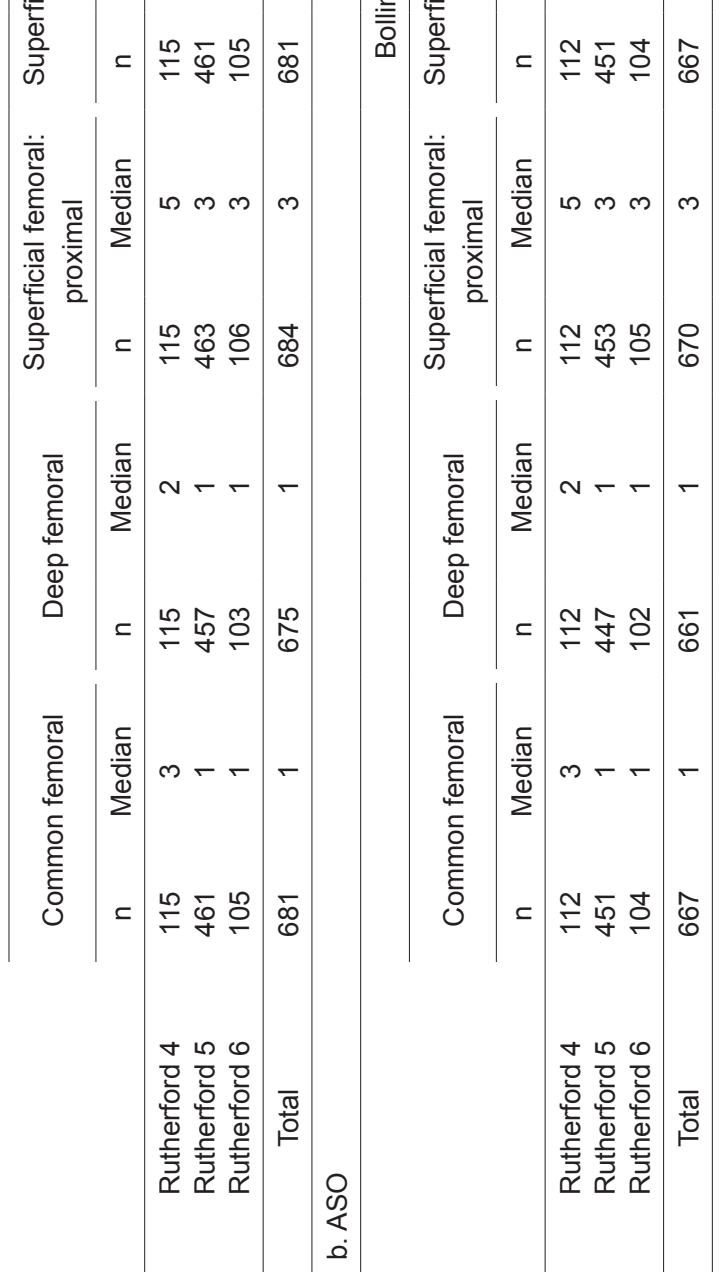


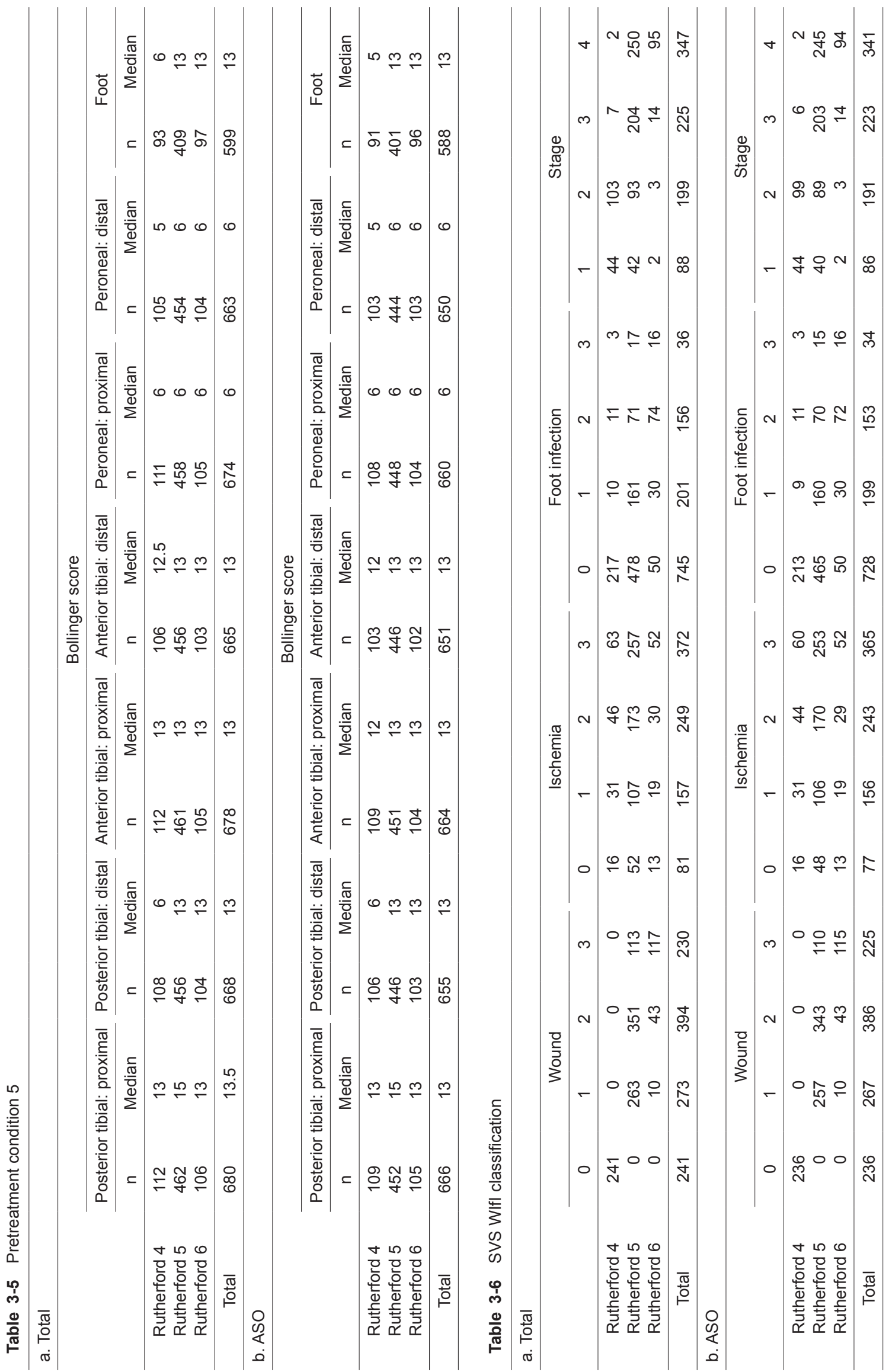




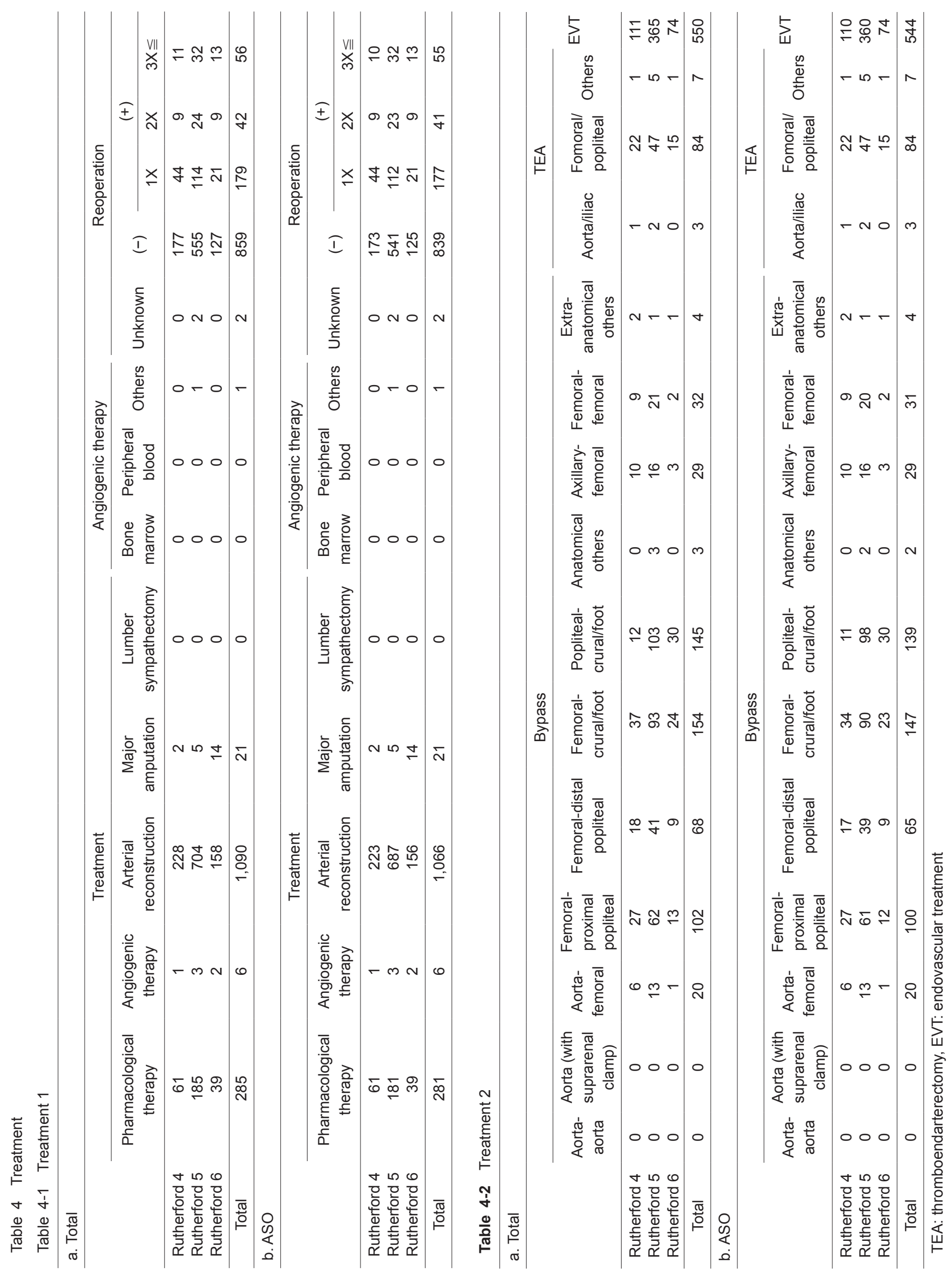




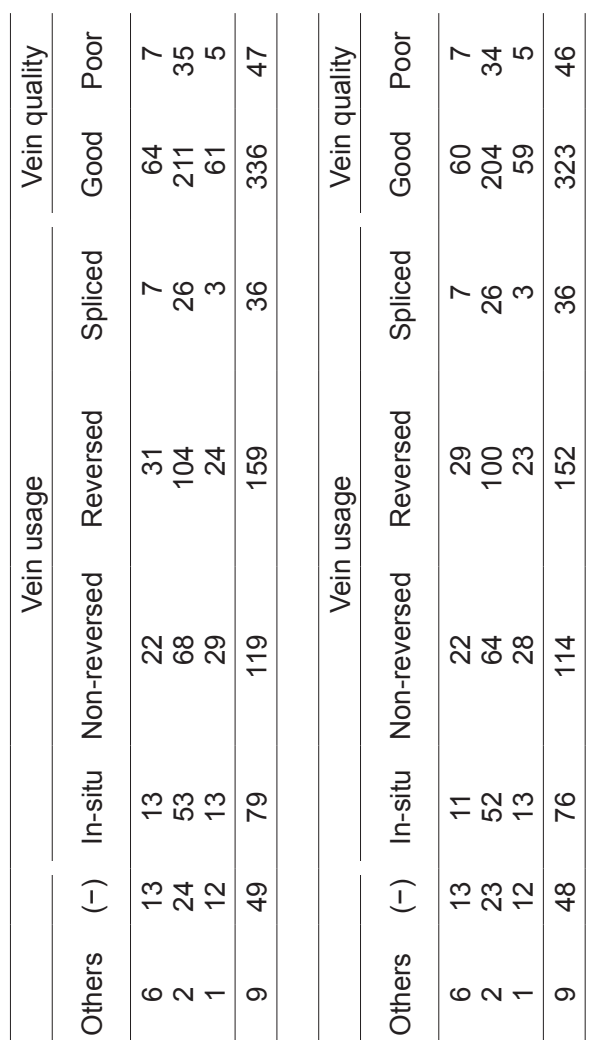

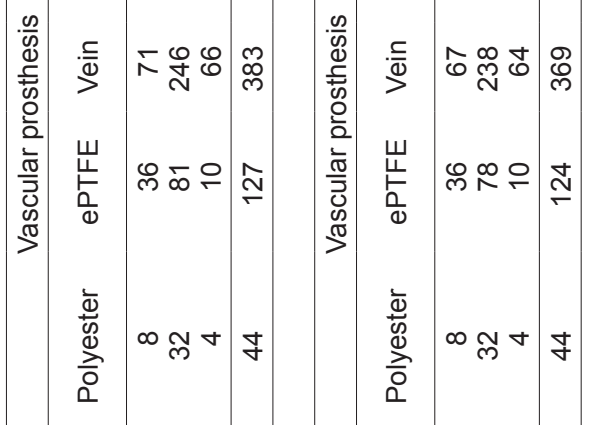

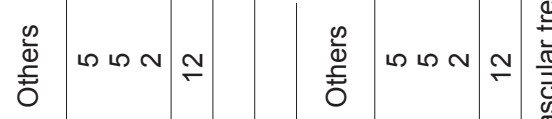

స్

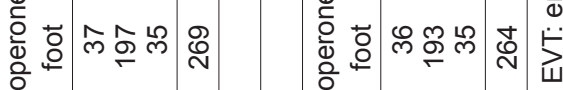

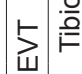
岀

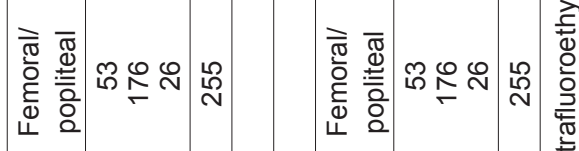

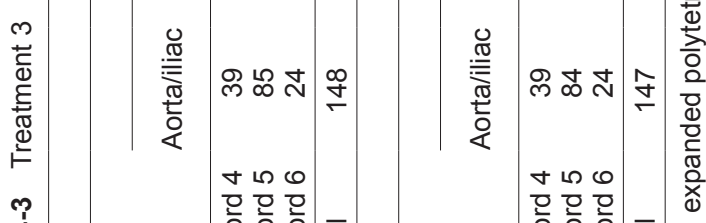

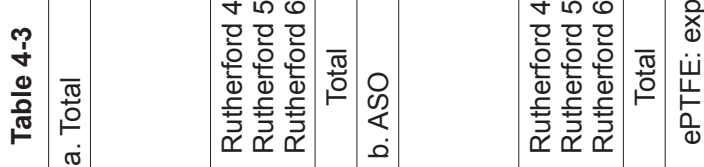

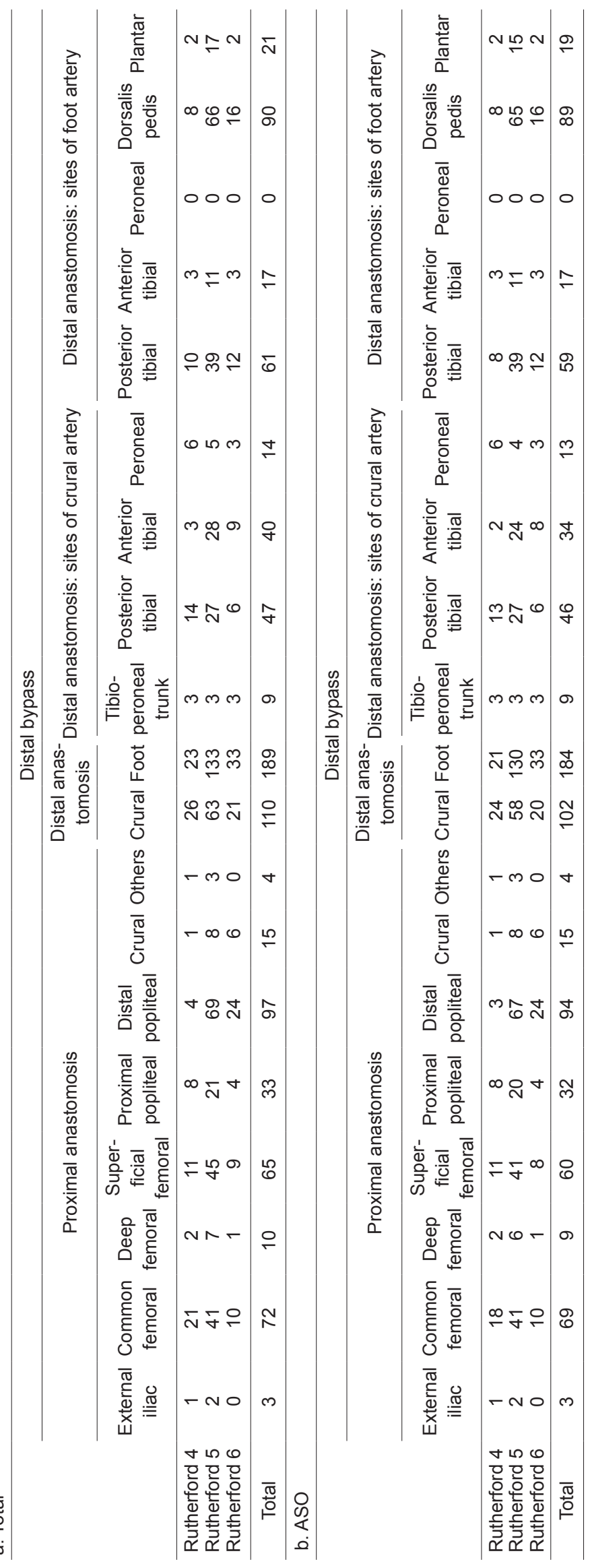




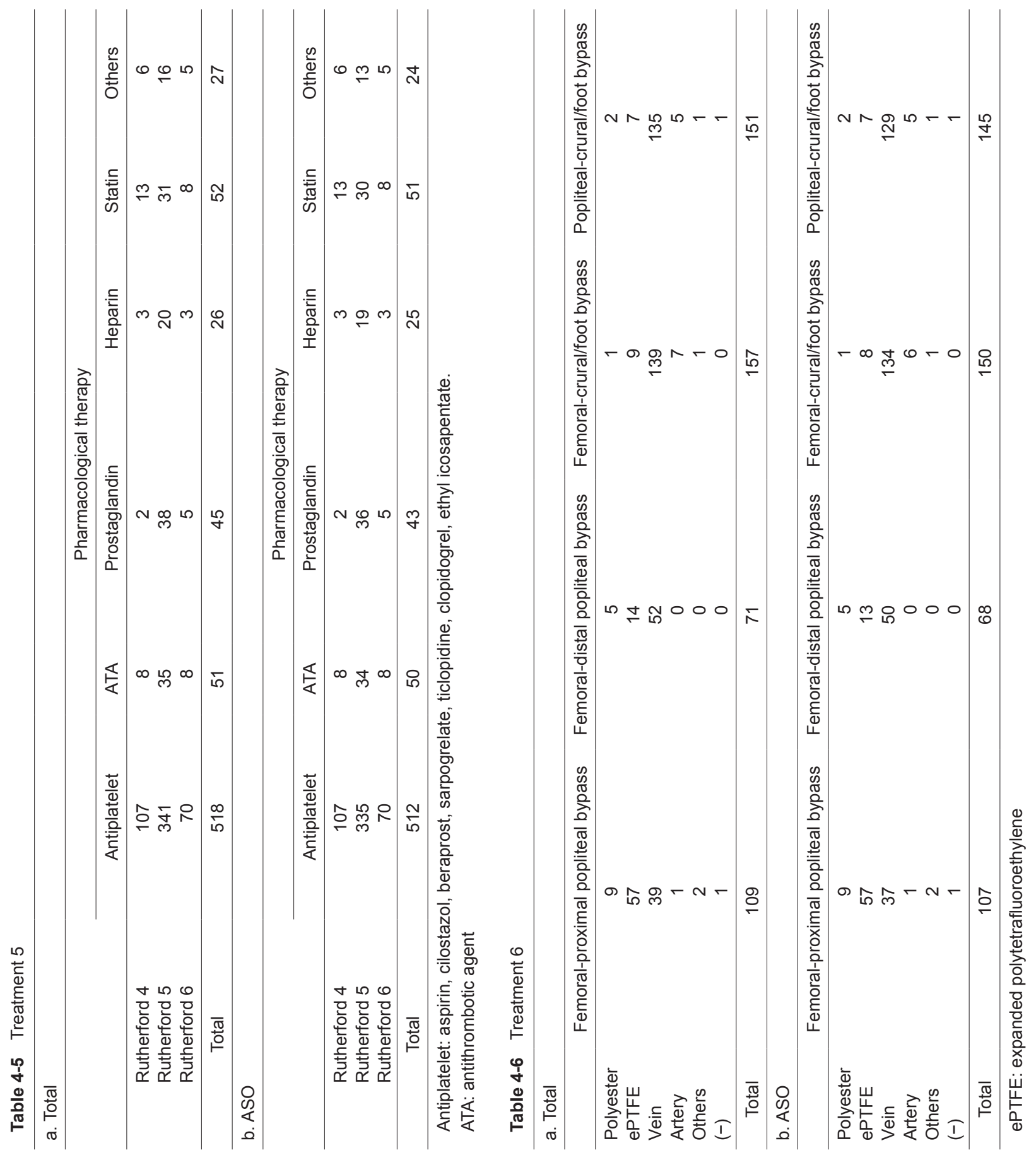




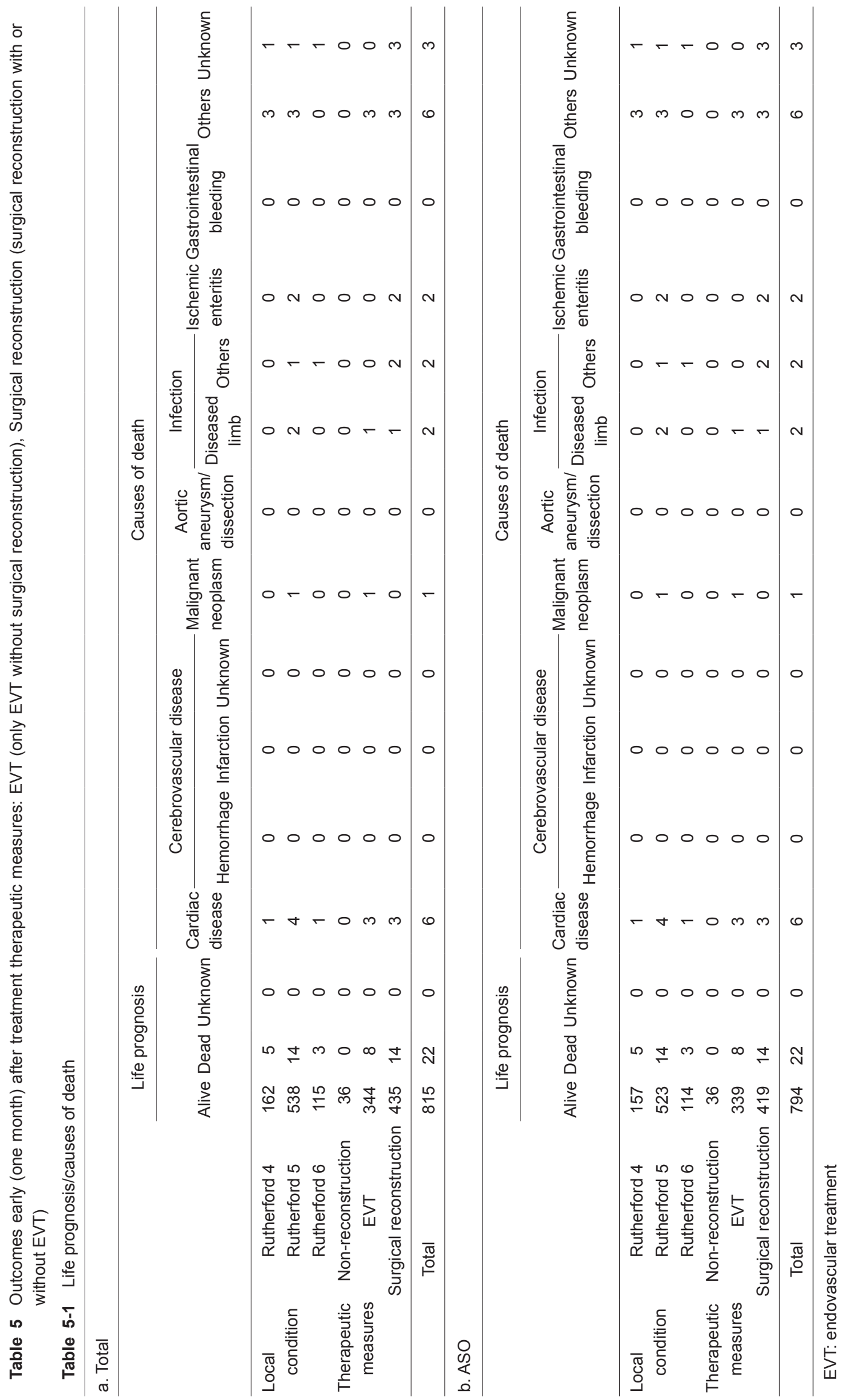




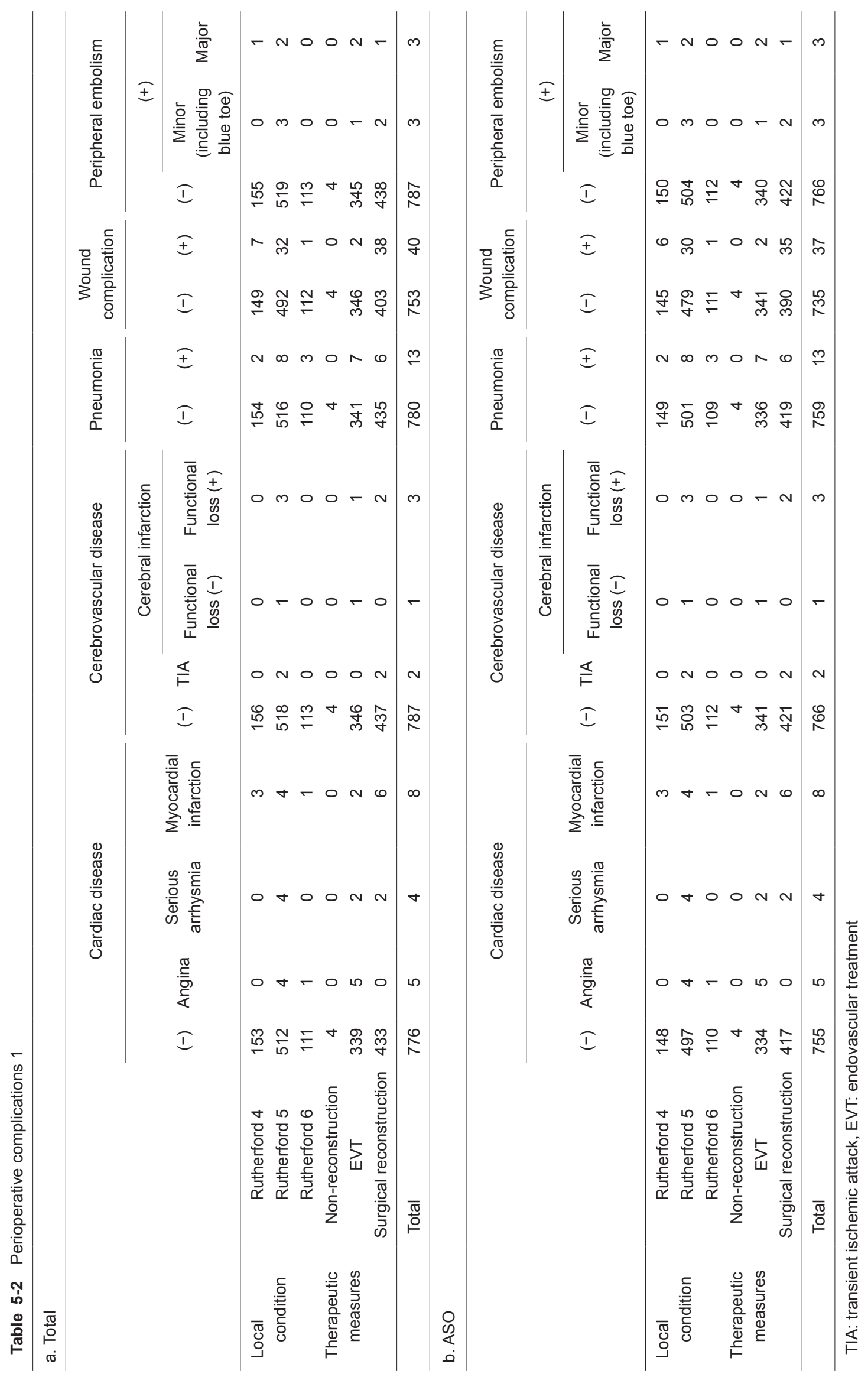




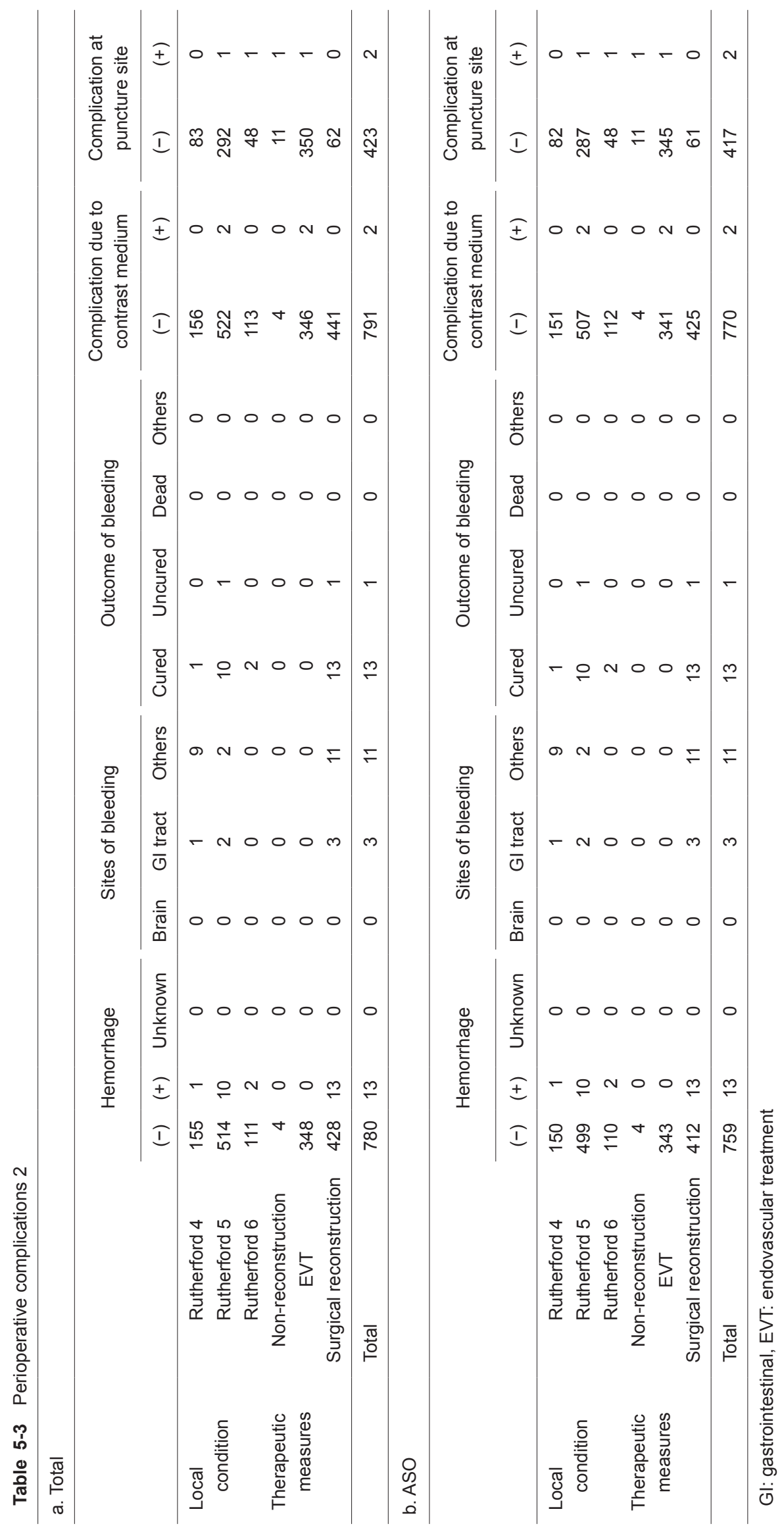




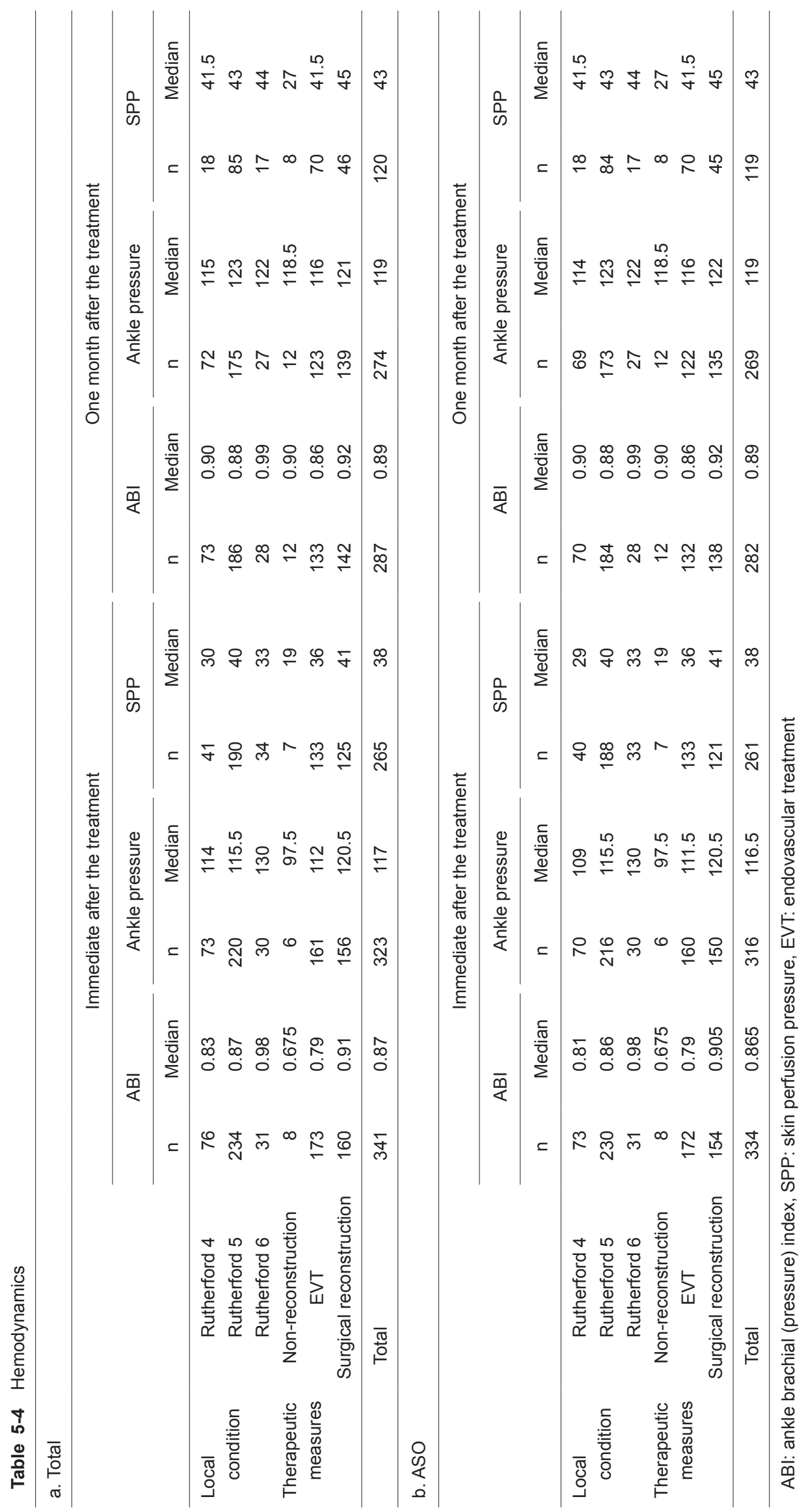




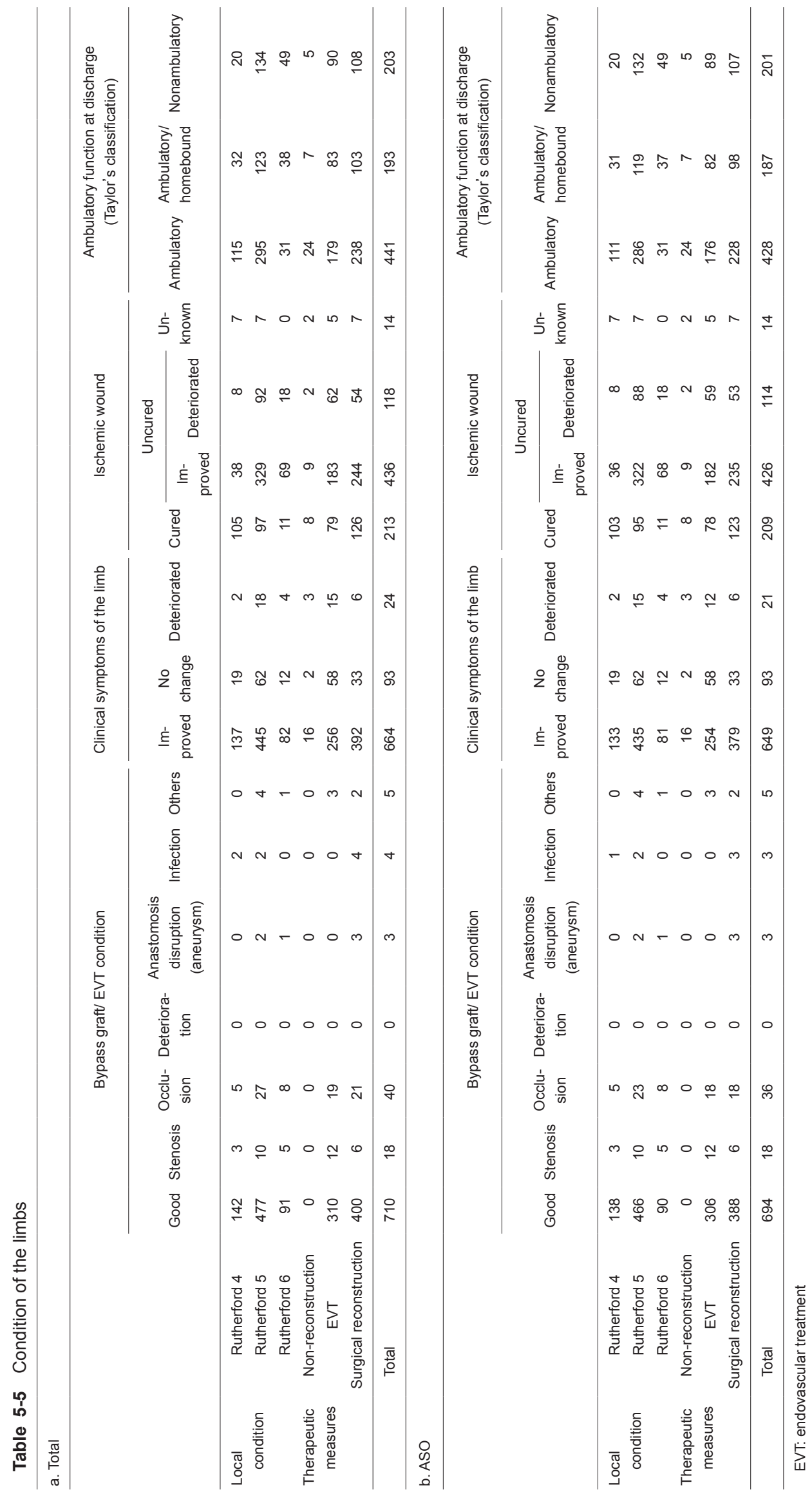




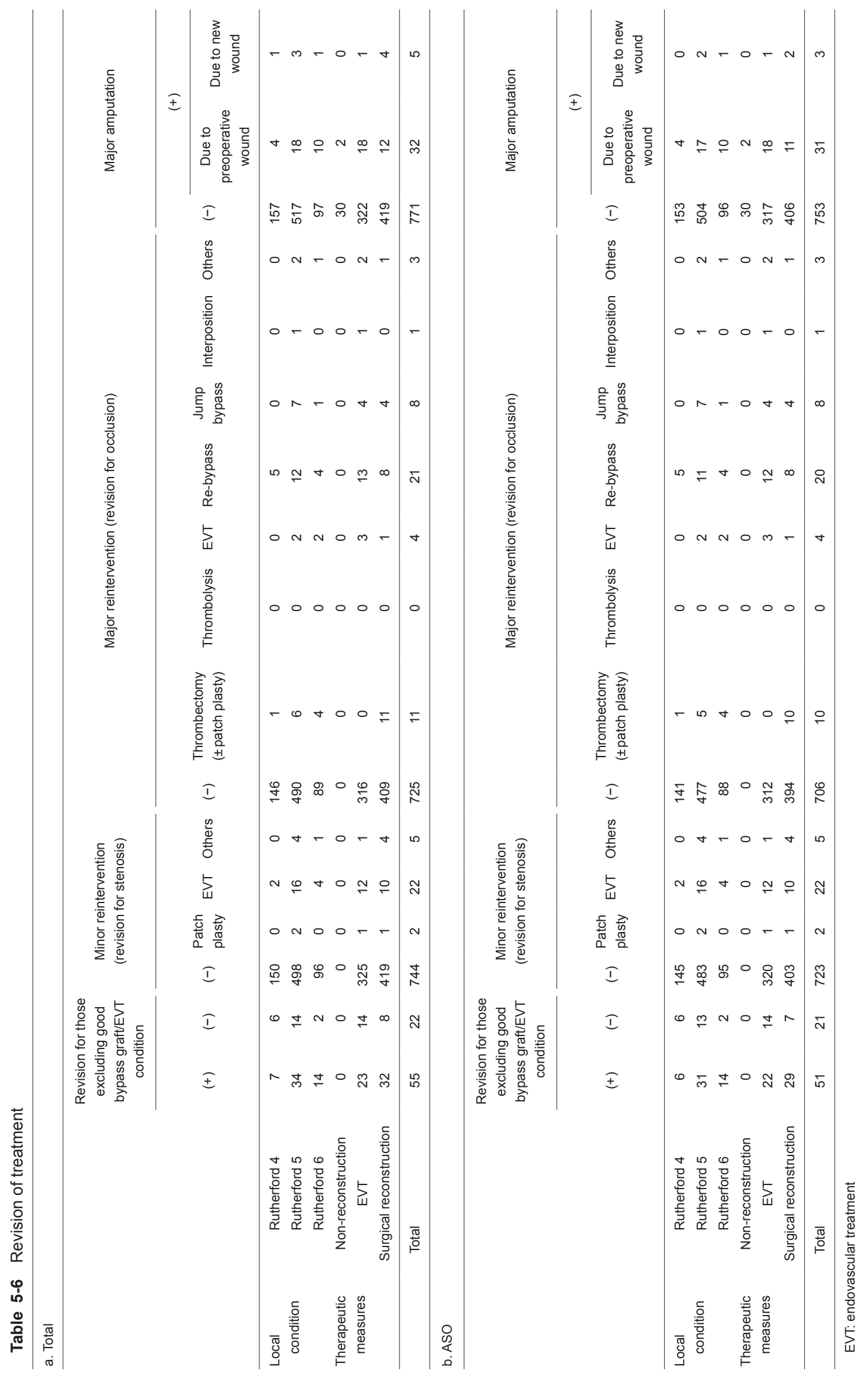




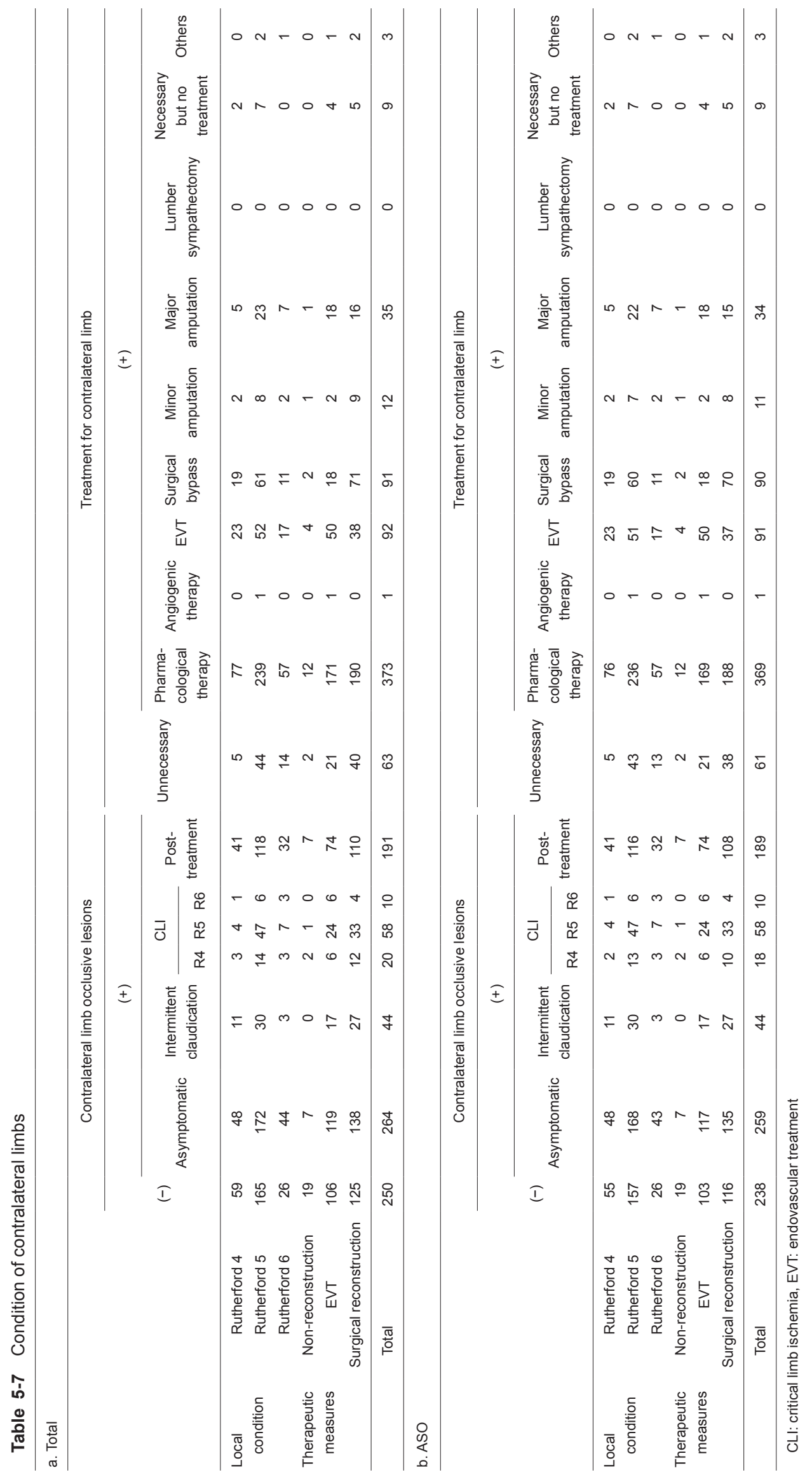




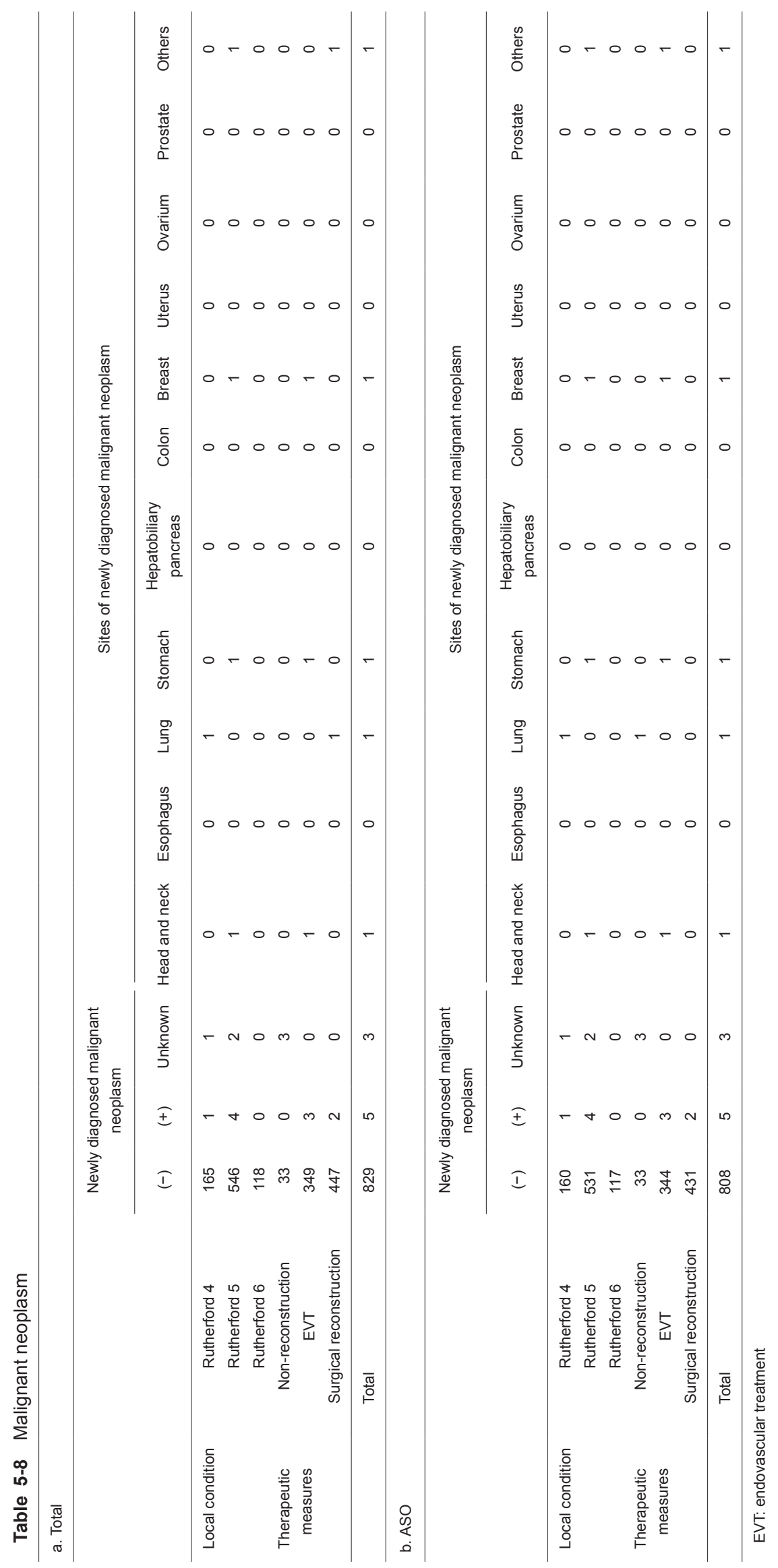

